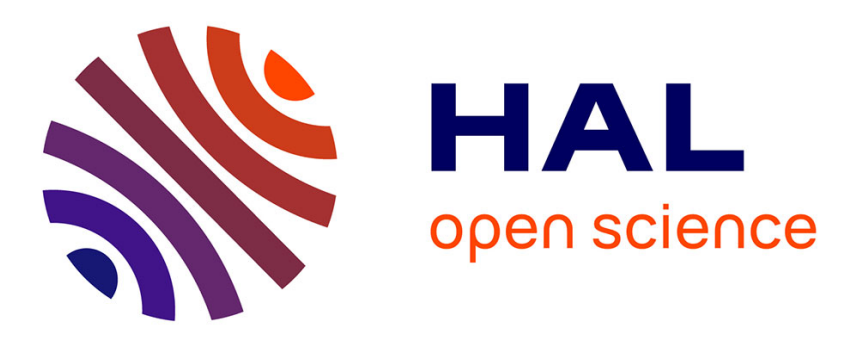

\title{
Single-file diffusion of particles in a box: Transient behaviors
}

\author{
Jean-Baptiste Delfau, Christophe Coste, Michel Saint-Jean
}

\section{To cite this version:}

Jean-Baptiste Delfau, Christophe Coste, Michel Saint-Jean. Single-file diffusion of particles in a box: Transient behaviors. Physical Review E: Statistical, Nonlinear, and Soft Matter Physics, 2012, 85, pp.61111 - 61111. 10.1103/PhysRevE.85.061111 . hal-01404836

\section{HAL Id: hal-01404836 \\ https://hal-univ-paris.archives-ouvertes.fr/hal-01404836}

Submitted on 29 Nov 2016

HAL is a multi-disciplinary open access archive for the deposit and dissemination of scientific research documents, whether they are published or not. The documents may come from teaching and research institutions in France or abroad, or from public or private research centers.
L'archive ouverte pluridisciplinaire HAL, est destinée au dépôt et à la diffusion de documents scientifiques de niveau recherche, publiés ou non, émanant des établissements d'enseignement et de recherche français ou étrangers, des laboratoires publics ou privés. 


\title{
Single-file diffusion of particles in a box: Transient behaviors
}

\author{
Jean-Baptiste Delfau, Christophe Coste, and Michel Saint Jean \\ Laboratoire Matière et Systèmes Complexes, Unité Mixte de Recherche, Centre National de la Recherche Scientifique 7057, \\ Université Paris Diderot, and PRES Sorbonne Paris Cité, \\ Bâtiment Condorcet, 10 rue Alice Domon et Léonie Duquet, 75205 Paris Cedex 13, France
}

(Received 21 February 2012; revised manuscript received 3 May 2012; published 11 June 2012)

\begin{abstract}
We consider a finite number of particles with soft-core interactions, subjected to thermal fluctuations and confined in a box with excluded mutual passage. Using numerical simulations, we focus on the influence of the longitudinal confinement on the transient behavior of the longitudinal mean squared displacement. We exhibit several power laws for its time evolution according to the confinement range and to the rank of the particle in the file. We model the fluctuations of the particles as those of a chain of springs and point masses in a thermal bath. Our main conclusion is that actual system dynamics can be described in terms of the normal oscillation modes of this chain. Moreover, we obtain complete expressions for the physical observables, in excellent agreement with our simulations. The correct power laws for the time dependency of the mean squared displacement in the various regimes are recovered, and analytical expressions of the prefactors according to the relevant parameters are given.
\end{abstract}

DOI: 10.1103/PhysRevE.85.061111

PACS number(s): 05.40.-a, 66.10.cg, 47.57.eb

\section{INTRODUCTION}

Diffusion of particles in a one-dimensional (1D) system where mutual exchanges are forbidden has been largely explored in the case of infinite systems with hard-core (or zero range) interactions between the particles, in the thermodynamic limit [1-10]. In this single-file diffusion (SFD), after an initial ballistic motion with a mean square displacement (MSD) scaling as $t^{2}$, the particles undergo a subdiffusive motion characterized by a MSD increasing as $t^{1 / 2}$. This non-Fickian $t^{1 / 2}$ scaling, which can be observed at long times, has also been predicted for particles with soft-core (or finite range) interactions [11-14].

A subdiffusive regime is observed for systems with periodic boundary conditions too [13,15-23]. For hard-core interactions, the $t^{1 / 2}$ regime is eventually followed by a linear diffusion at very long times. This corresponds to the free diffusion of the whole system considered as a unique effective particle with the overall mass $[6,16]$, and with a diffusion coefficient $D_{\text {tot }}$. For soft-core interactions, the earliest simulations showed that the MSD varies instead as $t^{1 / 2+\epsilon}$ where the small correction $\epsilon$ depends upon the kind of interparticle interaction $[24,25]$ in agreement with our own experimental results [20-22]. More recently, we exhibited more complex behaviors according to the number of particles and to the damping coefficient [13]. Indeed, after the initial ballistic motion, the MSD may exhibit three different regimes: a linear behavior growing as $D t$ where $D$ is a collective diffusion constant $\left(D \neq D_{\text {tot }}\right)$, a subdiffusive SFD behavior scaling as $F t^{1 / 2}$ where $F$ is a collective mobility constant, or these two behaviors in a sequence, as $D t$ then as $F t^{1 / 2}$. For low damping and a small number of particles, a ballistic behavior of the effective particle, growing as $t^{2}$, can be observed after the SFD regime and before its eventual diffusive scaling [13].

Those behaviors have been accounted for by describing the system of interacting particles as a chain of identical point masses linked by linear springs to their neighbors $[13,26]$. The chain motion is then described by the superposition of all the normal modes of oscillation. We have shown that the parts of the MSD due to the overdamped and underdamped modes respectively scale as $t^{1 / 2}$ and $t$, the relative amount of those two parts depending upon the damping coefficient. Moreover, the translational invariance of the system, described by a null frequency mode, induces at very long times the ballistic motion of the effective particle with a MSD $\propto t^{2}$ and, eventually, its diffusive motion with a MSD $\propto t$.

Finite linear systems have been seldom explored. Only a few experimental studies [27] and no complete theory are devoted to the SFD for particles with soft-core interactions in a finite channel $[6,28,29]$. In a previous paper [27], we have presented experimental results performed with a system of millimetric metallic balls, with soft-core screened electrostatic interactions. This system is put in an effective thermal bath insured by a random mechanical vibration [20-22]. Various regimes have been identified for the MSD time evolution. After an initial ballistic free motion $\left(t<t_{\text {corr }}\right)$, the particles undergo an intermediate subdiffusive motion $\left(t_{\mathrm{corr}}<t<t_{\text {sat }}\right)$ until the eventual saturation regime $\left(t_{\text {sat }}<t\right)$ where the MSD reaches its constant asymptotic value due to the finite system size.

Another important difference with respect to the cyclic systems is the loss of translational invariance. In finite linear systems, the diffusivities, the mobilities, and the crossover times $\left(t_{\text {corr }}\right.$ and $\left.t_{\text {sat }}\right)$ strongly depend upon the rank of the particle in the line. We have shown that the outermost particles reach their MSD saturation values always faster than the other particles (see Fig. 6 of Ref. [27]) and that the longest saturation times $t_{\text {sat }}$ are always associated to the inner balls. This is somewhat paradoxical since the latter are the more confined particles and are submitted to the strongest interparticle forces; we should therefore expect the quickest saturation for these particles. The actual observation of the opposite effect suggests a collective behavior due to long-ranged correlations.

We have undertaken numerical simulations to extend our previous study towards damping constants that are experimentally out of reach, and to easily control the longitudinal confinement. In a first paper [30], we have studied the saturation regime at asymptotically long times, $t_{\text {sat }} \ll t$. We have 
classified the observed behaviors according to the parameters $\lambda_{w}$ and $E_{w}$, respectively defined as the length scale and the magnitude of the confinement force. We have calculated a curve in the plane $\left(\lambda_{w}, E_{w}\right)$ that separates short-ranged from long-ranged confining forces. For short-ranged confinement, only the motions of the outermost particles are influenced by the confining walls, and the MSD saturation values decrease from the center particle down to the outermost ones with a roughly parabolic shape. For long-ranged confinement, the MSD saturation values do not vary monotonously with the particle index. On the contrary, it reaches symmetric minimal values for particles located between the walls and the center. When the confining force of each wall extends in the whole cell, this minimum takes place at the central particle whereas, quite surprisingly, the higher fluctuations are exhibited by the outermost particles. The confinement enhances the diffusion [30]. The dynamics is accounted for by the description as a chain of identical point masses linked by linear springs to their neighbors and to the confining walls, just as cyclic systems. All the long time behaviors of the particles are quantitatively recovered from a normal mode analysis of the chain.

In this paper, we focus on the influence of the confinement force on the transient regimes $\left(0<t<t_{\text {sat }}\right)$ of interacting particles confined in a box. We show that the time evolution of the MSD strongly depends on the characteristics of the confinement potential and on the rank of the particles in the line. The results of our simulations are analyzed with the same model used for the saturation regime analysis. In particular, we show that for each particle $n$, only a few normal modes contribute to the MSD and that the damping constant compared to the normal frequencies determines the time evolution of the MSD. We then deduce complete expressions for the physical observables, which provide an excellent agreement with our simulations.

The paper is organized as follows. In Sec. II A, we briefly describe our system and the simulation methods. In Sec. II B, we sum up the classification obtained in Ref. [30] for the confining force. Section III is devoted to the description of the dynamical regimes observed in our simulations (Sec. III A), of the crossover times that define the transitions between these regimes (Sec. III B), and to an unevenness that is a characteristic of finite-size systems when they reach saturation (Sec. III C). In Sec. IV, we interpret our observations with a masses and springs chain model. The conclusion (Sec. V) sums up our results.

\section{PARTICLES IN A FINITE BOX}

\section{A. Simulations}

We consider $(2 N-1)$ identical point particles of mass $M$ located in the $x-y$ plane, in a thermal bath at temperature $T$. The particles are confined by a quadratic potential in $y$ in such a way that they stay in the vicinity of the cell axis and cannot cross each other, as if they were diffusing in a narrow channel of finite length $L[13,30]$. Let $\mathbf{r}_{n}=\left(x_{n}, y_{n}\right)$ be the position of the particle $n$. The particle is submitted to the lateral confinement force $-\beta y_{n} \mathbf{e}_{y}$ of stiffness $\beta$, to the repulsive confinement forces $F_{w}\left(x_{n}\right)$ and $F_{w}\left(L-x_{n}\right)$ from the walls and to the interaction force $\mathbf{F}_{\text {int }}\left(\mathbf{r}_{n}\right)$ from the other particles. The confinement force is characterized by an energy scale $E_{w}$ and a length scale $\lambda_{w}$, and the interaction force by an energy scale $E_{0}$ and a length scale $\lambda_{0}$. We describe the dynamics with the Langevin equation

$$
\begin{aligned}
& M \ddot{\mathbf{r}}_{n}+M \gamma \dot{\mathbf{r}}_{n}-\mathbf{F}_{\text {int }}\left(\mathbf{r}_{n}\right) \\
& \quad-\left[F_{w}\left(x_{n}\right)-F_{w}\left(L-x_{n}\right)\right] \mathbf{e}_{x}-\beta y_{n} \mathbf{e}_{y}=\boldsymbol{\mu}(n, t)
\end{aligned}
$$

with $\gamma$ the damping constant and with $\mu(n, t)$ the random force applied on the particle $n$ at time $t$. Details of the model and of the algorithm may be found in Ref. [30].

The stiffness $\beta$ is such that $\left\langle\Delta y^{2}\right\rangle \ll\left\langle\Delta x^{2}\right\rangle$. The system dynamics thus always fulfills the SFD requirement. All simulations are done with a system of 33 interacting particles $(N=$ 17) confined in a channel of length $L=60 \mathrm{~mm}$, and a length scale $\lambda_{0}=0.48 \mathrm{~mm}$. These values have been selected in order to compare the simulations with our previous experiments [27]. The dimensionless mean density $L /\left(2 N \lambda_{0}\right)$ is of the same order of magnitude as in other numerical $[24,25,31]$ and experimental [15,17-19,32-35] studies. In Ref. [30], we have modified the particle number in the range $8 \leqslant N \leqslant 66$ at constant mean density, and obtained convincing indications that the behavior of systems with $N=17$ particles is generic. The temperature $T$ and the amplitude $E_{0}$ are such that the dimensionless parameter $E_{0} /\left(k_{B} T\right) \approx 5$, which is also of the same order of magnitude as in other studies. The results obtained with 33 interacting particles are thus generically relevant to the SFD in a box.

\section{B. The longitudinal confinement}

Three domains must be considered according to the values of the parameters $\lambda_{w}$ and $E_{w}$ [30]. The area of short-ranged (SR) confinement is separated in the plane $\left(\lambda_{w}, E_{w}\right)$ from the area of long-ranged (LR) confinement (see Fig. 7 of Ref. [30]). The separation curve is not a power law, but the rough estimate $E_{w} / E_{0}=\left(2 \lambda_{0} / \lambda_{w}\right)^{1.22}$ may be useful. All points $\left(\lambda_{w}, E_{w}\right)$ above this estimate corresponds to a long-ranged confinement. It is noteworthy that the area of long-ranged confinement is not defined by the characteristic length alone.

The SR domain, for which the influence of each confining wall extends over the nearest particle only, may be itself separated in hard wall (HW) and soft wall (SW) domains according to the relative importance of the confinement with respect to the interparticle interactions. If we model the system as a chain of identical point masses linked by linear springs to their neighbors and to the confining walls, as in Sec. IV A, all the springs describing the interparticle interactions have the same stiffness $k$ whereas the stiffness $k_{w}$ describing the outermost particles interaction with the walls is smaller (higher) than $k$ in the SW (HW) case [30]. For LR confinement, the springs' stiffness depends upon the rank of the particles.

We have the parameters $\lambda_{w}$ and $E_{w}$ vary by two decades. In their respective natural scales, they range in the intervals $\lambda_{w} \in\left[\lambda_{0}, 60 \lambda_{0}\right]$ and $E_{w} \in\left[0.005 E_{0}, 0.1 E_{0}\right]$. For the sake of comparison, the mean interparticle distance is $L /(2 N)=$ $3.68 \lambda_{0}$ and the cell length is $125 \lambda_{0}$. The three different kinds of confinement have been studied in our simulations. For SR confinement, the HW $\left(k_{w}>k\right)$ and SW $\left(k_{w}<k\right)$ cases respectively correspond to $\left(\lambda_{w}=0.48 \mathrm{~mm}, E_{w} / E_{0}=510^{-2}\right)$ and $\left(\lambda_{w}=1 \mathrm{~mm}, E_{w} / E_{0} \in\{0.1,0.5,1\}\right)$ respectively. For LR 
confinement, we consider either a constant length $\lambda_{w}=15$ $\mathrm{mm}$ with $E_{w} / E_{0}$ between $510^{-3}$ and 0.5 or a constant $E_{w} / E_{0}=0.1$ and $\lambda_{w}$ between $4 \mathrm{~mm}$ and $30 \mathrm{~mm}$.

\section{SIMULATION RESULTS}

When the system is in thermal equilibrium, the thermal fluctuations induce small displacements $u_{n}$ of the particles around their equilibrium positions $x_{n}^{*}$, such that $x_{n}(t)=$ $x_{n}^{*}+u_{n}(t)$. The instantaneous MSD of the particle $n$ is written $\left\langle\Delta u(n, t)^{2}\right\rangle$.
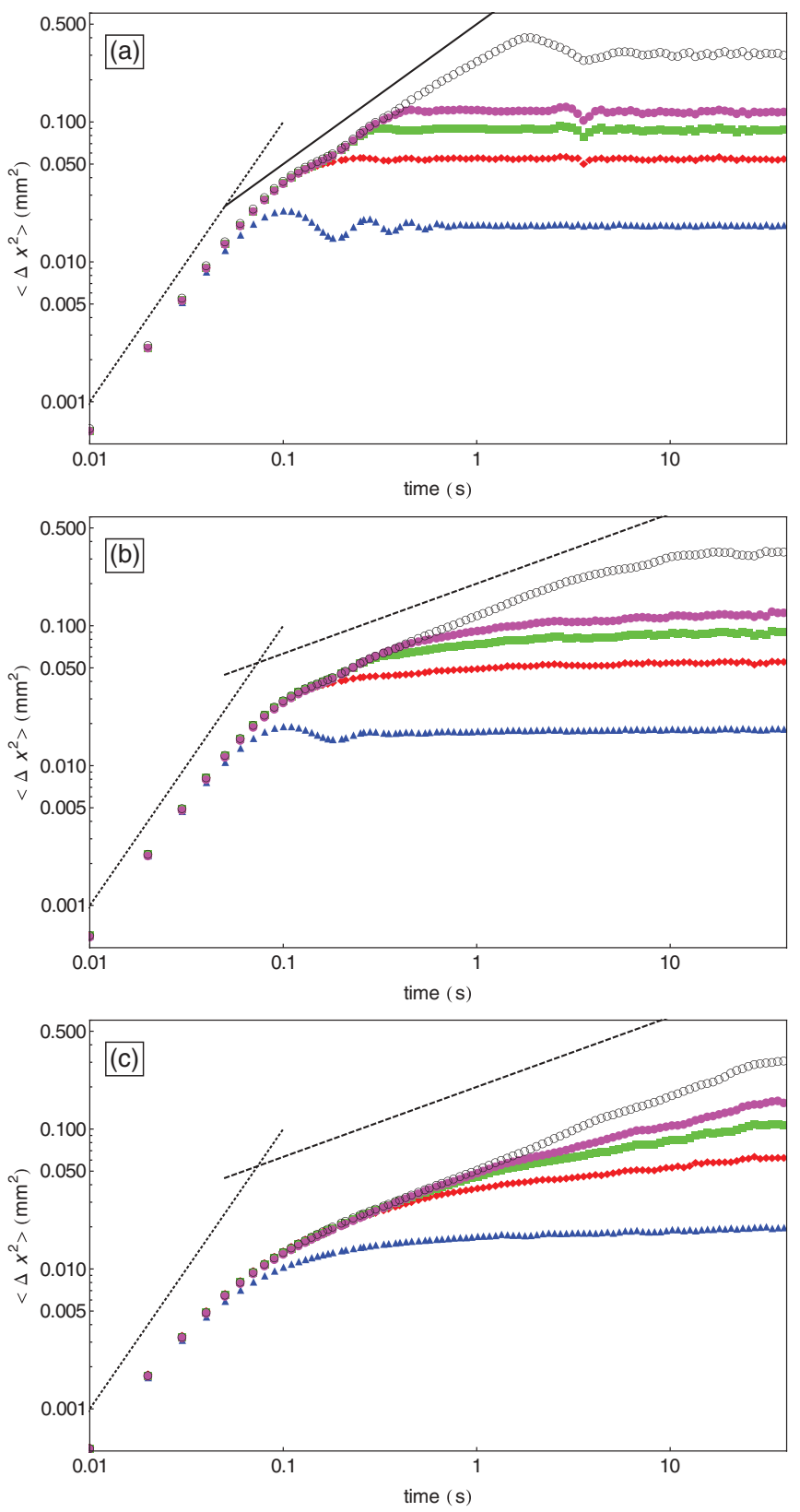

\section{A. Intermediate regime $\left(t_{\text {corr }}<t<t_{\text {sat }}\right)$}

A ballistic regime with $\left\langle\Delta u(n, t)^{2}\right\rangle \propto t^{2}$ is observed when the damping is not too large at times $t<t_{\text {corr }}$. In the intermediate regime, three different behaviors with peculiar power laws can be distinguished according to the damping, the particle index, and the kind of confinement. The MSD may saturate right after the ballistic regime. It may rather exhibit stretched oscillations around either a linear time scaling, a square root time scaling, or both behaviors successively. These evolutions strongly depend on the kind of confinement.

Let us begin with SR confining force, in the HW case. In Fig. 1, we plot $\left\langle\Delta u(n, t)^{2}\right\rangle$ for the four outer particles, $13 \leqslant$
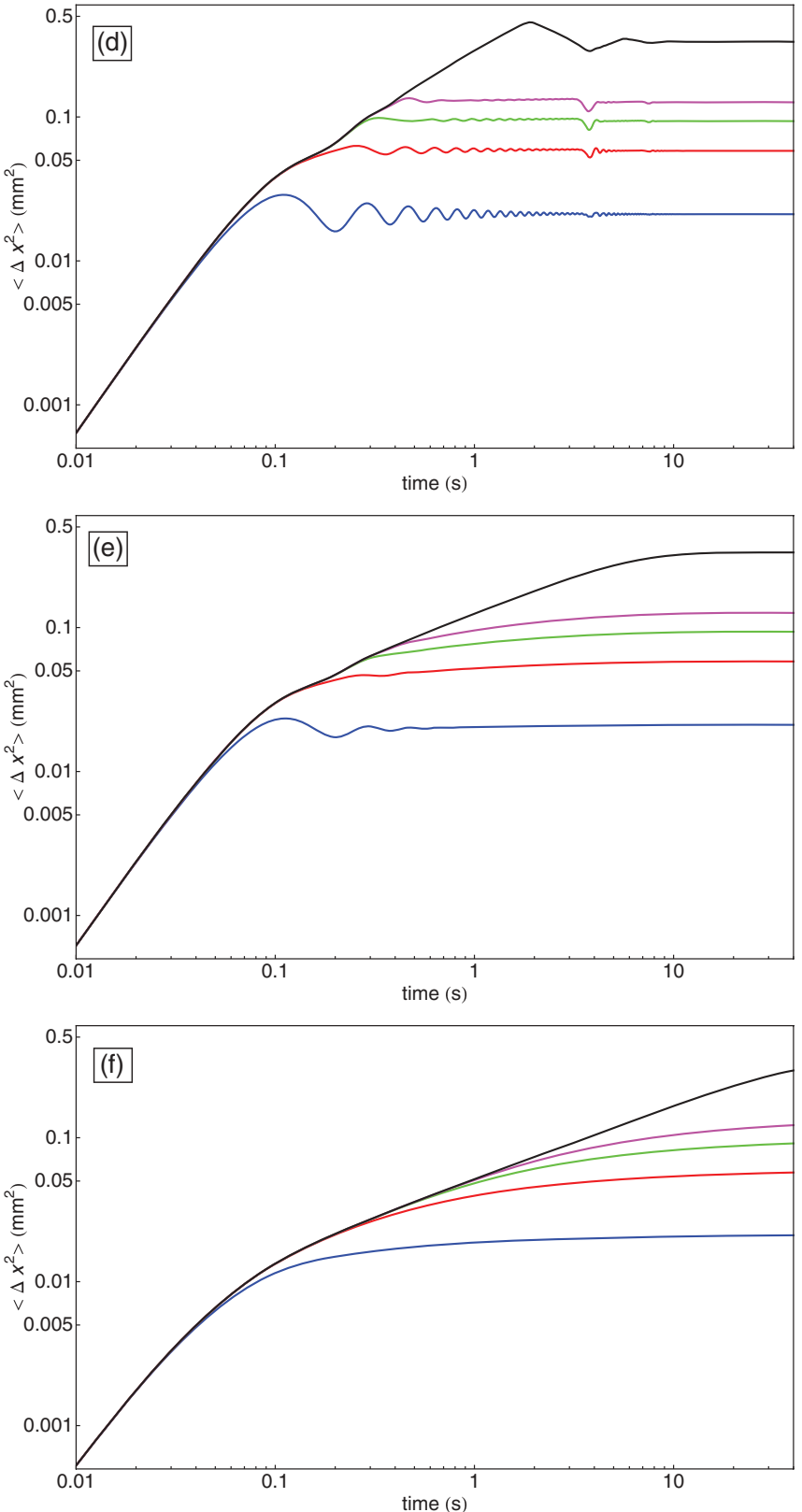

FIG. 1. (Color online) Plot of MSD $\left\langle\Delta u(n, t)^{2}\right\rangle$ (in $\mathrm{mm}^{2}$ ) as a function of time (in s), for a system of 33 particles and HW confinement with $\lambda_{w}=0.48 \mathrm{~mm}$ and $E_{w}=0.0095 E_{0}$. We show the particles with $n= \pm 16$ (blue dots), $n= \pm 15$ (red squares), $n= \pm 14$ (green diamonds), $n= \pm 13$ (magenta triangles up), and $n=0$ (black triangles down): $\gamma=1 \mathrm{~s}^{-1}$ [(a) and (d)]; $\gamma=10 \mathrm{~s}^{-1}$ [(b) and (e)]; $\gamma=60 \mathrm{~s}^{-1}$ [(c) and (f)]. Left column: simulations data; right column: calculations. Both axes in Log scales. Dotted lines of slope 2, solid lines of slope 1, and dashed lines of slope $1 / 2$. 
TABLE I. Diffusivities and mobilities of the inner particles in Fig. 2.

\begin{tabular}{|c|c|c|c|c|c|}
\hline \multicolumn{2}{|c|}{$\gamma=1 \mathrm{~s}^{-1}$} & \multicolumn{2}{|c|}{$\gamma=60 \mathrm{~s}^{-1}$} & \multicolumn{2}{|c|}{$E_{w} / E_{0}=0.1$} \\
\hline$E_{w} / E_{0}$ & $D_{\mathrm{SW}}\left(\mathrm{mm}^{2} \mathrm{~s}^{-1}\right)$ & $E_{w} / E_{0}$ & $F_{\mathrm{SW}}\left(\mathrm{mm}^{2} \mathrm{~s}^{-1 / 2}\right)$ & $\gamma\left(\mathrm{s}^{-1}\right)$ & $F_{\mathrm{SW}}\left(\mathrm{mm}^{2} \mathrm{~s}^{-1 / 2}\right)$ \\
\hline 0.1 & 0.155 & 0.01 & 0.050 & 10 & 0.11 \\
\hline 0.5 & 0.140 & 0.05 & 0.036 & 20 & 0.08 \\
\hline 1 & 0.135 & 1 & 0.030 & 60 & 0.045 \\
\hline
\end{tabular}

$n \leqslant 16$, and the central one $n=0$. The MSD of the outermost particle behaves with a strikingly specific time dependence. For small damping $\left(\gamma=1 \mathrm{~s}^{-1}\right),\left\langle\Delta u(16, t)^{2}\right\rangle$ exhibits a direct transition from the ballistic regime to a regime of damped oscillations around its saturation value at long times. These oscillations, not observed for the other particles, decrease
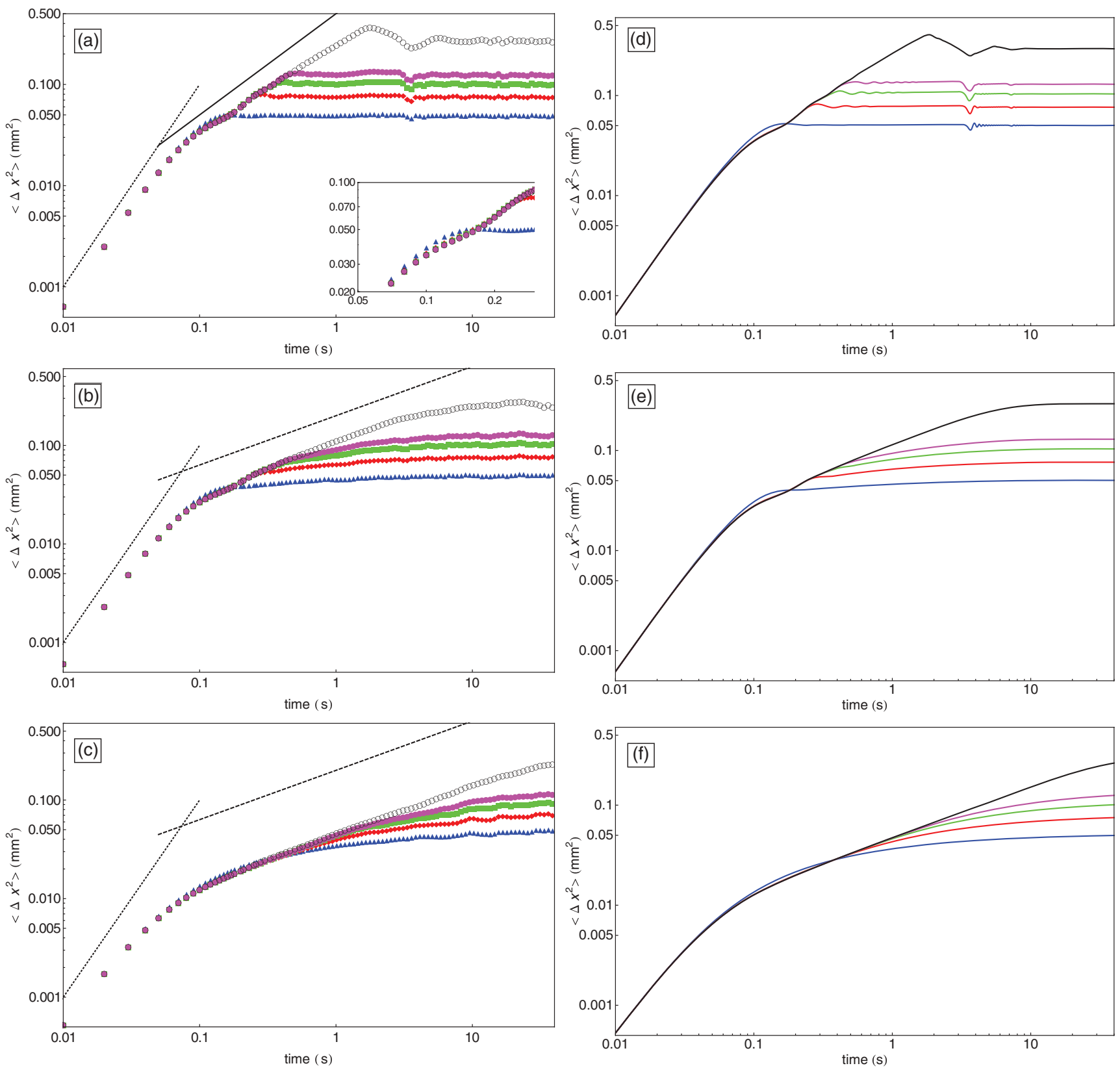

FIG. 2. (Color online) Plot of $\operatorname{MSD}\left\langle\Delta u(n, t)^{2}\right\rangle$ (in $\mathrm{mm}^{2}$ ) as a function of time (in s), for a system of 33 particles and for a SW confining potential with $\lambda_{w}=1 \mathrm{~mm}$ and $E_{w}=0.1 E_{0}$. We show the particles with $n= \pm 16$ (blue dots), $n= \pm 15$ (red squares), $n= \pm 14$ (green diamonds), $n= \pm 13$ (magenta triangles up), and $n=0$ (black triangles down). The damping is $\gamma=1 \mathrm{~s}^{-1}\left[(\mathrm{a})\right.$ and (d)]; $\gamma=10 \mathrm{~s}^{-1}[(\mathrm{~b})$ and (e)]; $\gamma=60 \mathrm{~s}^{-1}$ [(c) and (f)]. Left column: simulations data; right column: calculations. Both axes in Log scales. Dotted lines of slope 2, solid lines of slope 1 , and dashed lines of slope $1 / 2$. In the inset we zoom on the curves crossing. 

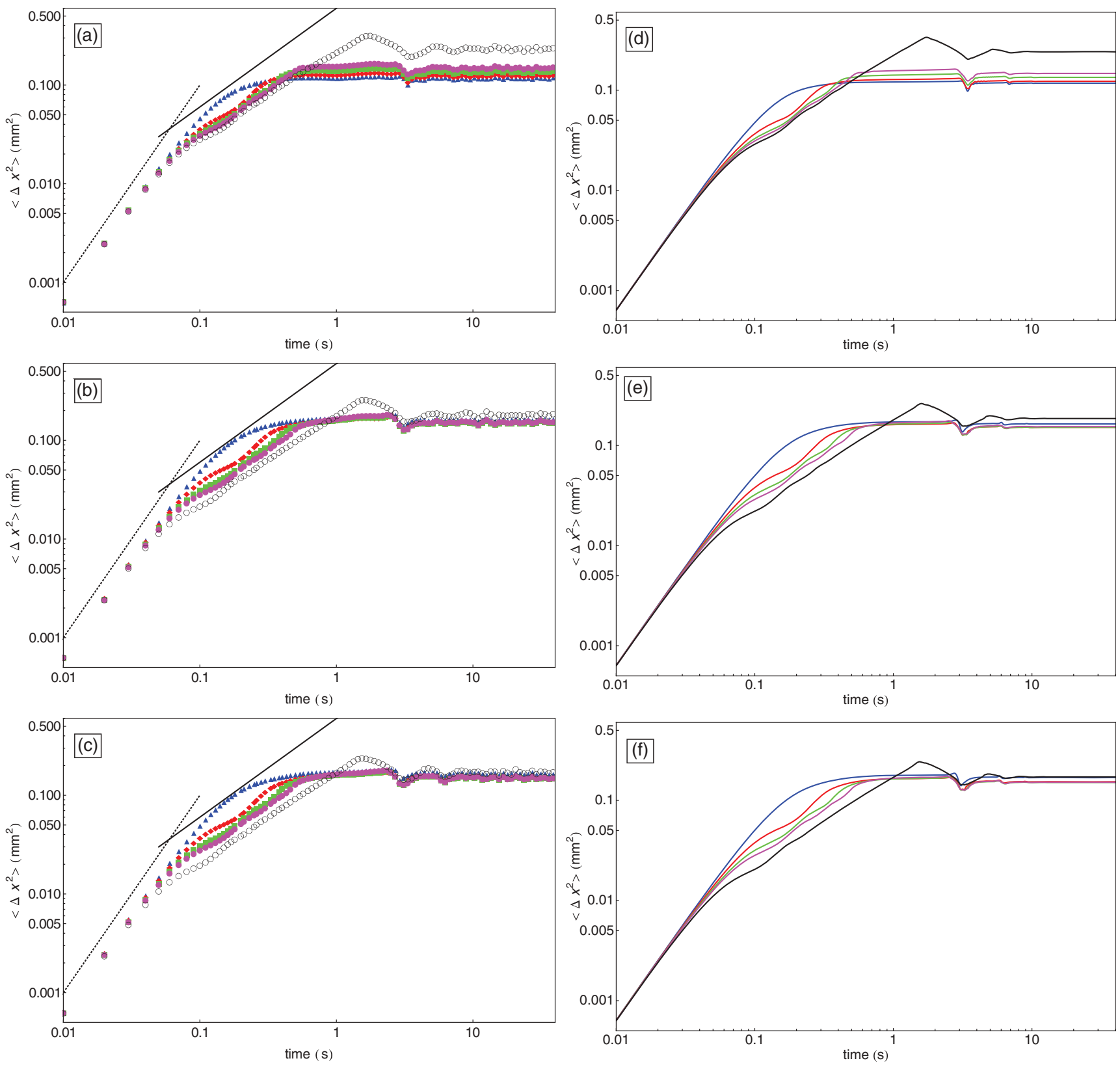

FIG. 3. (Color online) Plot of MSD $\left\langle\Delta u(n, t)^{2}\right\rangle$ (in $\mathrm{mm}^{2}$ ) as a function of time (in s), for a system of 33 particles and a LR confining potential with $E_{w}=0.1 E_{0}$ and $\lambda_{w}=4 \mathrm{~mm} \mathrm{[(a)} \mathrm{and} \mathrm{(d)],} \lambda_{w}=11 \mathrm{~mm} \mathrm{[(b)} \mathrm{and} \mathrm{(e)],} \mathrm{and} \lambda_{w}=30 \mathrm{~mm}\left[(\mathrm{c})\right.$ and (f)]. The damping is $\gamma=1 \mathrm{~s}^{-1}$. We show the particles with $n= \pm 16$ (blue dots), $n= \pm 15$ (red squares), $n= \pm 14$ (green diamonds), $n= \pm 13$ (magenta triangles down), and $n=0$ (black triangles up). Left column: simulations data; right column: calculations. Both axes in Log scales. Dotted lines of slope 2, solid lines of slope 1 .

with the damping and are almost suppressed for $\gamma=60 \mathrm{~s}^{-1}$. All other particles display the same intermediate regime, as shown by the superposition of the MSD plots before their respective saturation [see Figs. 1(a)-1(c)]. For small damping $\left[\gamma=1 \mathrm{~s}^{-1}\right.$, see Fig. 1(a)], the MSD is roughly linear in time until its saturation. A single diffusion coefficient $D_{\mathrm{HW}}$ can be determined for all particles. It is smaller than the single-particle free diffusivity $D_{0}\left(D_{\mathrm{HW}}=0.16 \mathrm{~mm}^{2} \mathrm{~s}^{-1}, D_{0}=6.4 \mathrm{~mm}^{2} \mathrm{~s}^{-1}\right)$, which proves that this Fickian diffusion is a collective behavior of the system. When the damping increases, we observe an SFD scaling $\left\langle\Delta u(n, t)^{2}\right\rangle=F_{\mathrm{HW}} t^{1 / 2}$ with roughly the same mobility $F_{\mathrm{HW}}$ for all particles [see Figs. 1(b)-1(c)]. Its typical value is $F_{\mathrm{HW}}=0.12 \mathrm{~mm}^{2} \mathrm{~s}^{-1 / 2}$ for $\gamma=60 \mathrm{~s}^{-1}$.

We now consider SR confining force, in the SW case. Comparing Fig. 2 and Fig. 1, we see that the main difference concerns the outermost particle. Its MSD does not oscillate anymore, but presents the fastest evolution towards saturation. The characteristic feature of SW confinement is thus the crossing between the MSD plot of the outermost particle and those of the other particles. In contrast, the inner particles still present the same intermediate regime before their respective saturation, as in the HW case [see Figs. 2(a)-2(c)]. We may 

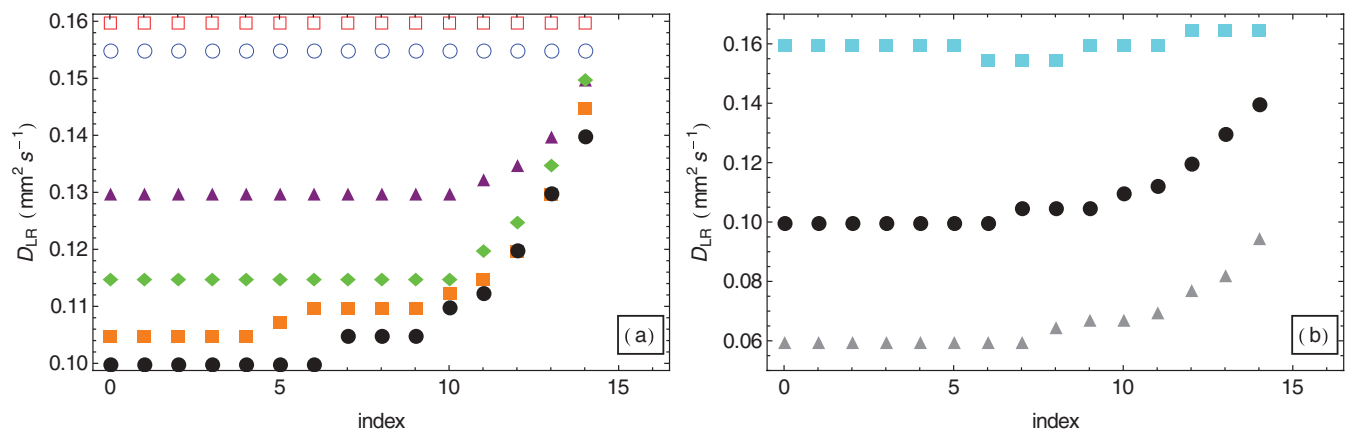

FIG. 4. (Color online) Diffusivities $\left(\mathrm{mm}^{2} \mathrm{~s}^{-1}\right.$ ) as a function of the particle index for 33 particles and $\gamma=1 \mathrm{~s}^{-1}$. (a) HW confinement, $\lambda_{w}=$ $0.48 \mathrm{~mm}, E_{w} / E_{0}=0.0095$ (red open squares); SW confinement, $\lambda_{w}=0.48 \mathrm{~mm}, E_{w} / E_{0}=0.1$ (blue circles); LR confinement, $E_{w} / E_{0}=0.1$, $\lambda_{w}=4 \mathrm{~mm}$ (magenta triangles), $7 \mathrm{~mm}$ (green diamonds), $11 \mathrm{~mm}$ (orange squares), $15 \mathrm{~mm}$ (black dots). (b) LR confinement, $\lambda_{w}=15 \mathrm{~mm}$, $E_{w} / E_{0}=0.005$ (cyan squares), 0.1 (black dots), 0.5 (gray triangles).

define either a diffusivity or a mobility for the inner particles. Both decrease very weakly with increasing amplitude $E_{w}$, as shown in Table I. The mobility may be defined only at large damping. As shown in the last column of Table I, the mobilities strongly decrease with the damping. Note that these values are very close to those measured in the HW case. We shall return to this point in the next section.

For LR confining forces, the intermediate regime depends on the particle index. The outermost particles evolve from the ballistic to the saturated regime directly as in the previous case. On the contrary, the inner particles undergo a slowing down of their dynamics that is more and more significant the farther they are from the walls. The superposition of the MSD curves is recovered only in the central part of the cell. Moreover, the differences between particles become more pronounced the further we are in the LR area of the $\left(\lambda_{w}, E_{w}\right)$ plane (Fig. 3). Similar behaviors are observed if $E_{w}$ is increased with $\lambda_{w}$ fixed to a large value. These effects are observable whatever the damping.

The diffusivity $D_{\mathrm{LR}}\left(\lambda_{w}, E_{w}, n\right)$ decreases with the particle distance from the wall over a distance roughly equal to the confinement length $\lambda_{w}$ [see Fig. 4(a)]. The further we are in the LR area, the larger the difference between the diffusivities of the outermost and inner particles. We underline that whatever $\lambda_{w}$ and $E_{w}$, these diffusivities are always smaller than for a free particle $\left(D_{0}=12.8 \mathrm{~mm}^{2} \mathrm{~s}^{-1}\right)$ with the same damping and temperature.

For larger dampings, the time evolution of the MSD of the inner particles exhibits the SFD scaling $\left\langle\Delta u(n, t)^{2}\right\rangle \propto$ $t^{1 / 2}$ (see Fig. 5). A mobility $F_{\mathrm{LR}}\left(\lambda_{w}, E_{w}, n\right)$ may thus be defined as was the case for the diffusivities $D_{\mathrm{LR}}\left(\lambda_{w}, E_{w}, n\right)$. We plot in Fig. 6 its evolution according to the particle index for several confining force configurations. We see that going further in the $\mathrm{LR}$ region of the $\left(\lambda_{w}, E_{w}\right)$ plane increases the number of particles that exhibit a SFD behavior, decreases their mobility, with the smaller mobility for the inner particles at a given $\left(\lambda_{w}, E_{w}\right)$. Moreover, the mobilities also decrease with the damping whatever the particle. All these results are in qualitative agreement with those of previous experiments [27].

\section{B. Crossover times}

The correlation times $t_{\text {corr }}$ exhibit some systematic behaviors (see Fig. 7). For the SR case, $t_{\text {corr }}$ is roughly constant, except for the outermost particles for which it is either one third smaller or higher respectively in the HW and SW cases. On the contrary, in the LR case, the correlation times progressively increase when the particle gets closer to the confinement wall, over a distance roughly equal to confinement length [see Fig. 7(a)]. Let us emphasize that its value near the edge is now twice higher than the values for the inner particles, which are of the same order of magnitude than those measured in the SR cases. On the other hand, the correlation time decreases with the confinement magnitude $E_{w}$ [see Fig. 7(b)]. Lastly, these times remain independent of the damping for small values of $\gamma$ but strongly decrease when the damping coefficient $\gamma$ increases.
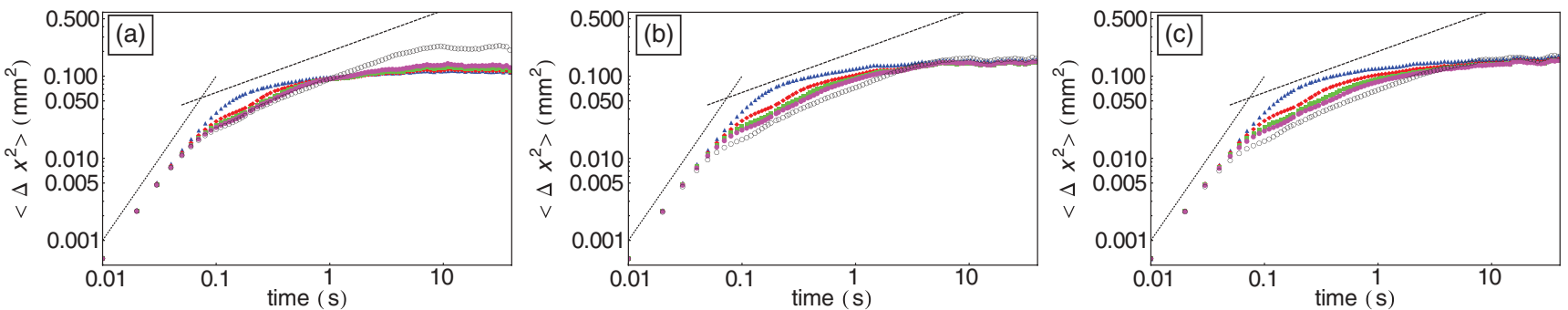

FIG. 5. (Color online) Plot of MSD $\left\langle\Delta u(n, t)^{2}\right\rangle$ (in $\mathrm{mm}^{2}$ ) as a function of time (in s), for a system of 33 particles and a LR confining potential with $E_{w}=0.1 E_{0}$ and $\lambda_{w}=4 \mathrm{~mm}(\mathrm{a}) ; 11 \mathrm{~mm}(\mathrm{~b}), 30 \mathrm{~mm}(\mathrm{c})$. The damping is $\gamma=10 \mathrm{~s}^{-1}$. We display the particles with $n= \pm 16$ (blue dots), $n= \pm 15$ (red squares), $n= \pm 14$ (green diamonds), $n= \pm 13$ (magenta triangles up), and $n=0$ (black triangles down). Dotted lines of slope 2 , and dashed lines of slope $1 / 2$ 

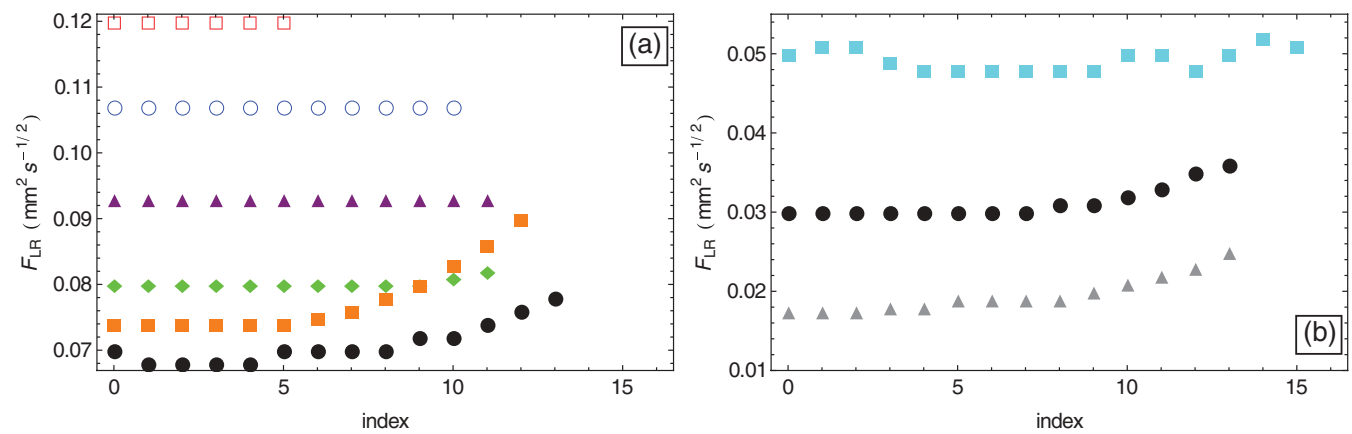

FIG. 6. (Color online) Mobility $\left(\mathrm{mm}^{2} \mathrm{~s}^{-1 / 2}\right.$ ) as a function of the particle index for 33 particles: (a) $\gamma=1 \mathrm{~s}^{-1}$. HW confinement, $\lambda_{w}=0.48$ $\mathrm{mm}, E_{w} / E_{0}=0.0095$ (red open squares); $\mathrm{SW}$ confinement, $\lambda_{w}=0.48 \mathrm{~mm}, E_{w} / E_{0}=0.1$ (blue circles); LR confinement, $E_{w} / E_{0}=0.1$, $\lambda_{w}=4 \mathrm{~mm}$ (magenta triangles), $7 \mathrm{~mm}$ (green diamonds), $11 \mathrm{~mm}$ (orange squares), $15 \mathrm{~mm}$ (black dots). (b) $\gamma=60 \mathrm{~s}^{-1}$. LR confinement, $\lambda_{w}=15 \mathrm{~mm}, E_{w} / E_{0}=0.001$ (red diamonds), 0.05 (pink circles), 0.1 (black dots).

After the intermediate regime, the saturation times $t_{\text {sat }}$ decrease roughly linearly according to the particle index $n$ (see Fig. 8). For small damping, the saturation times are independent of $\gamma$. As the damping increases, the $t_{\text {sat }}$ associated to the outer particles remains unchanged whereas those of the inner particles largely increase. The saturation times $t_{\text {sat }}$ for the inner and the outer particles evolve differently for a LR confinement. When we go further in the LR area, $t_{\text {sat }}$ decreases for the inner particles whereas the opposite is observed for the outer particles [see Figs. 8(b) and 8(c)]. In our simulations, the saturation times $t_{\text {sat }}$ are one to three orders of magnitude larger than the correlation times $t_{\text {corr }}$, in agreement with our experiments [27], whereas simulations for hard-core (zero ranged) interactions suggest five orders of magnitude between these two times [28,29]. This confirms the important influence of the finite (nonzero) range of the interactions on the particle dynamics.

\section{The unevenness at saturation}

Finally, let us indicate another characteristic feature of the finite-size systems at the beginning of the saturation regime. For small damping, we systematically observe at $t=t_{\text {un }}$ a little unevenness in the time evolution of $\left\langle\Delta u(n, t)^{2}\right\rangle$ in the saturation regime, which is otherwise parallel to the time axis [see Figs. 1(a) and 1(d), Figs. 2(a) and 2(d), and Fig. 3].
For given parameters $\left(\lambda_{w}, E_{w}\right)$, the time $t_{\mathrm{un}}$ is the same for all particles however the unevenness depth decreases as the particle gets closer to the wall. For the outermost particles, it is very small or completely lost. When $E_{w}$ is fixed, the unevenness is shifted towards short times as $\lambda_{w}$ increases. Similar behavior is observed when $\lambda_{w}$ is fixed and $E_{w}$ is raised (Fig. 3). This feature disappears at larger damping.

\section{MODELING AND INTERPRETATION}

\section{A. Normal modes analysis}

The small displacement $u(n, t)$ induced by the thermal fluctuations around the equilibrium position $x^{*}(n)$ is reasonably assumed such that $|u(n, t)| \ll d_{n}$, where $d_{n}$ is the distance between the particles $n$ and $n+1$. Using this approximation, we derive from Eq. (1) a model of a chain of $(2 N-1)$ identical point masses $M$ linked by linear springs to their neighbors and to both confining walls, submitted to dissipation and thermal fluctuations $[13,26]$. We therefore have to solve a set of coupled Langevin equations that reads

$$
\begin{aligned}
M \ddot{u}_{n}= & k_{n} u_{n-1}-\left(k_{n}^{w}+k_{n}+k_{n+1}\right) u_{n} \\
& +k_{n+1} u_{n+1}-M \gamma \dot{u}_{n}+\mu(n, t),
\end{aligned}
$$
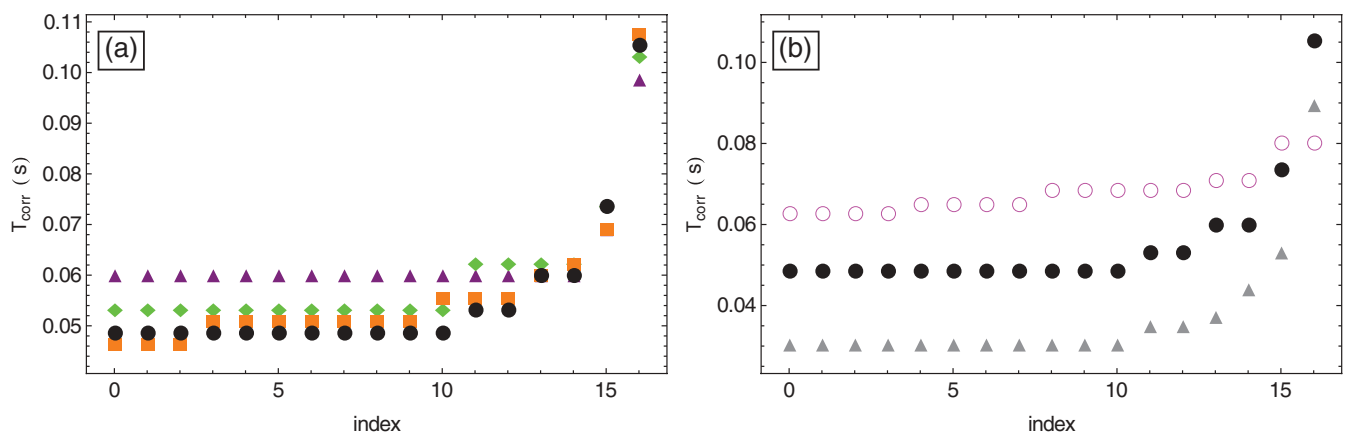

FIG. 7. (Color online) Correlation time $t_{\text {corr }}(\mathrm{s})$ as a function of the particle index for 33 particles, $\gamma=1 \mathrm{~s}^{-1}$ and a LR confinement. (a) $E_{w} / E_{0}=0.1, \lambda_{w}=4 \mathrm{~mm}$ (magenta triangles), $7 \mathrm{~mm}$ (green diamonds), $11 \mathrm{~mm}$ (black dots), $15 \mathrm{~mm}$ (orange squares). (b) $\lambda_{w}=15 \mathrm{~mm}$, $E_{w} / E_{0}=0.005$ (pink circles), 0.1 (black dots), 0.5 (gray triangles). 

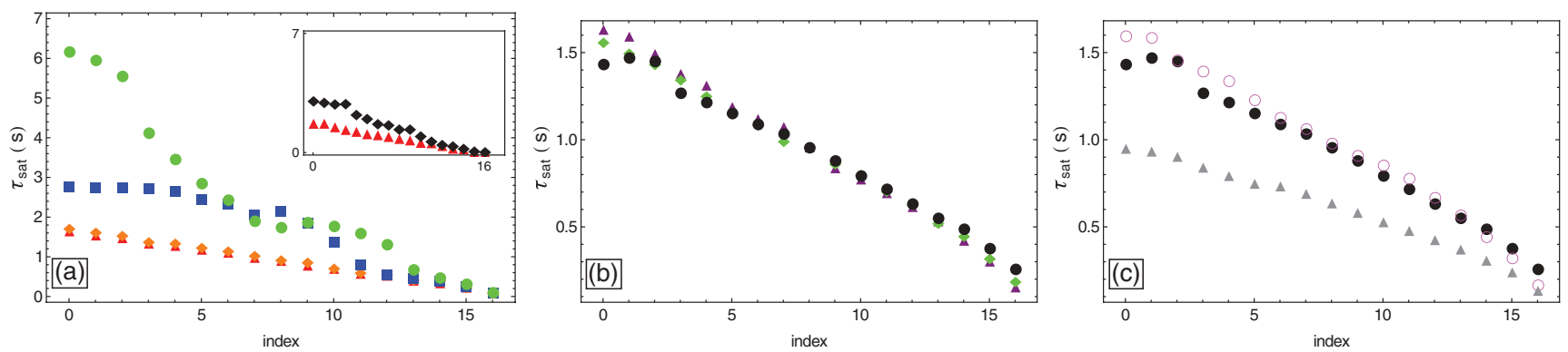

FIG. 8. (Color online) Saturation time $t_{\mathrm{sat}}$ (s) as a function of the particle index for 33 particles. (a) SW confinement, $E_{w} / E_{0}=0.1, \lambda_{w}=1$ $\mathrm{mm}: \gamma=0.1 \mathrm{~s}^{-1}$ (red triangles); $1 \mathrm{~s}^{-1}$ (orange diamonds); $10 \mathrm{~s}^{-1}$ (blue square); $20 \mathrm{~s}^{-1}$ (green dots). Inset: Plot of $t_{\mathrm{sat}}$ in the HW case: $\gamma=1$ $\mathrm{s}^{-1}$ (red triangles); $10 \mathrm{~s}^{-1}$ (black diamonds). (b) LR confinement, $E_{w} / E_{0}=0.1, \gamma=1 \mathrm{~s}^{-1}, \lambda_{w}=4 \mathrm{~mm}$ (magenta triangles); $7 \mathrm{~mm}$ (green diamonds); $15 \mathrm{~mm}$ (black dots). (c) LR confinement with $\gamma=1 \mathrm{~s}^{-1}, \lambda_{w}=15 \mathrm{~mm}, E_{w} / E_{0}=0.05$ (pink circles); 0.1 (black dots); 0.5 (gray triangles).

with $n \in[-N, N]$ and with $u(-N, t)=u(N, t)=0$. The spring stiffnesses are given by

$$
\begin{aligned}
k_{n} & \equiv-\frac{\partial F_{\mathrm{int}}}{\partial x}\left(d_{n}\right), \\
k_{n}^{w} & \equiv-\frac{\partial F_{w}}{\partial x}\left(L / 2+x_{n}^{*}\right)-\frac{\partial F_{w}}{\partial x}\left(L / 2-x_{n}^{*}\right) .
\end{aligned}
$$

For a set of confinement parameters $\left(\lambda_{w}, E_{w}\right)$, the equilibrium positions of the particles are measured on the simulations data. Then the spring stiffnesses are deduced from these positions since the expressions of the confining and interparticle force are known.

Some typical stiffness distributions are displayed in Fig. 9(a) (SR confinement, SW, and HW) and Fig. 9(b) (LR confinement). For the SR cases, all $k_{n}=k$ are the same, whereas each outermost particle is linked to its nearest wall by a spring of stiffness $k_{w}$. We consider two SR cases with roughly identical stiffness $k$, in order to identify the specific effects of the confinement, with $k_{w}(\mathrm{HW}) \approx 5 k_{w}(\mathrm{SW})$. In the LR case, the distribution of equilibrium positions induces complex dependencies of the stiffnesses $k_{n}^{w}$ and $k_{n}$ with $\lambda_{w}$ and $E_{w}$. The wall-particle $k_{n}^{w}$ remains always smaller than $k_{n}$ whatever the particle $n$ (the case $k_{n}^{w}>k_{n}$ is not as interesting as it looks like the HW case). Going further in the LR domain, the equilibrium positions of the outermost particles get further from the walls, which compresses the inner particles. This results in a large decrease of the wall-particle stiffnesses for the outermost particles and a moderate increase of the interparticle stiffnesses.

Knowing the stiffnesses, we write the sparse symmetric matrix, which is the characteristic matrix associated to the system of equations (2),

$$
S_{p n}=-k_{n} \delta_{p, n-1}+\left(k_{n}^{w}+k_{n}+k_{n+1}\right) \delta_{n, p}-k_{n+1} \delta_{p, n+1} .
$$

The characteristic frequencies $\omega_{s}$ are given by the eigenvalues $\sigma_{s}$ of $S_{p n}$, with $\omega_{s}^{2}=\sigma_{s} / M$. Several examples are presented in Fig. 10(a) for various confinements. The frequencies increase with the range of the confining force. In the $\mathrm{HW}$ case, the system has localized modes, which are characterized by high frequencies, larger than the cutoff frequency of a homogeneous chain with a mean stiffness $k(L / 2 N)$, as shown in Fig. 10(b) [30].

The normal modes $\mathbf{U}_{s}$ are the eigenvectors of this matrix, with components $U_{s}(n)$. The displacement $u(n, t)$ of the particle $n$ at time $t$ is expressed in terms of the normal modes as $u(n, t)=\sum_{s=1}^{2 N-1} U_{s}(n) X_{s}(t)$. Each amplitude $X_{s}(t)$ behaves as a damped oscillator forced by a random force, so that the MSD of each normal mode is

$$
\begin{aligned}
\left\langle\Delta X_{s}^{2}(t)\right\rangle & =\frac{2 k_{B} T}{M \omega_{s}^{2}}\left[1+\frac{\omega_{-}(s) e^{\omega_{+}(s) t}}{\omega_{+}(s)-\omega_{-}(s)}-\frac{\omega_{+}(s) e^{\omega_{-}(s) t}}{\omega_{+}(s)-\omega_{-}(s)}\right] \\
\omega_{ \pm}(s) & \equiv-\frac{\gamma}{2} \pm \sqrt{\frac{\gamma^{2}}{4}-\omega_{s}^{2}} .
\end{aligned}
$$
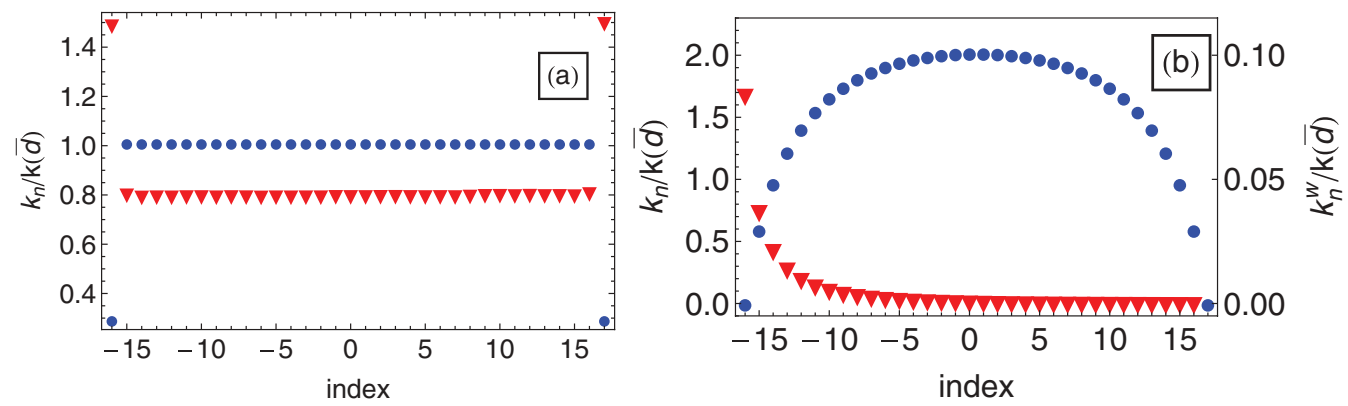

FIG. 9. (Color online) Dimensionless stiffness as a function of the particle index, for 33 particles. (a) SR confinement. Interparticle stiffness $k_{n} / k(\bar{d})$ for a SW (blue dots) and HW (red triangles). (b) LR confinement, $\lambda_{w}=11 \mathrm{~mm}, E_{w}=0.1 E_{0}$. Interparticle stiffness $k_{n} / k(\bar{d})($ left axis, blue dots) and confinement stiffness $-\frac{\partial F_{w}}{\partial x}\left(L / 2+x_{n}^{*}\right) / k(\bar{d})$ (right axis, red triangles). 

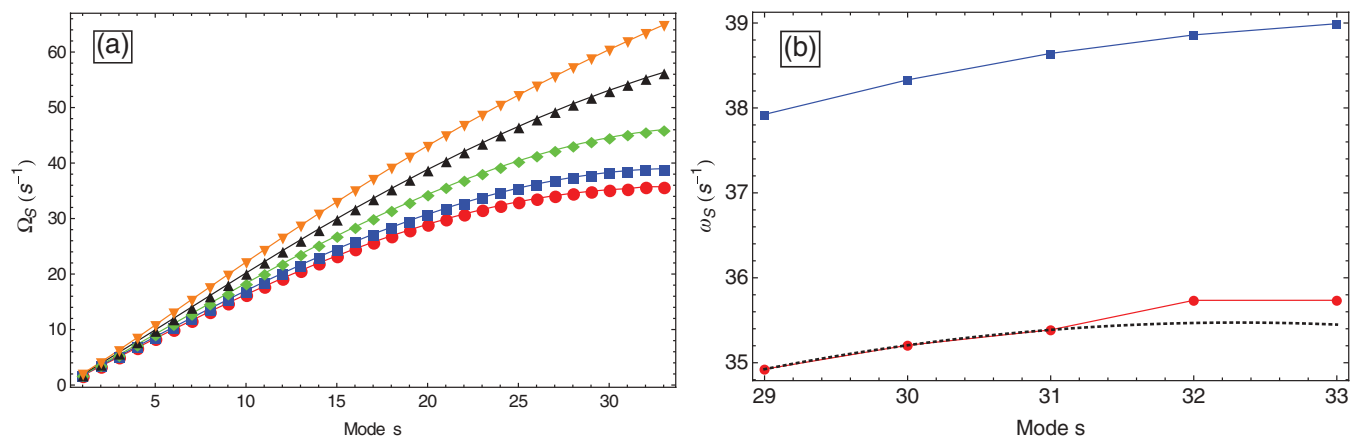

FIG. 10. (Color online) (a) Dispersion relation of the normal modes for a system of 33 particles. HW confinement, $\lambda_{w}=0.48$ mm, $E_{w} / E_{0}=0.0095$ (red dots); SW confinement, $\lambda_{w}=1 \mathrm{~mm}, E_{w} / E_{0}=0.1$ (blue squares); LR confinement, $E_{w} / E_{0}=0.1, \lambda_{w}=4 \mathrm{~mm}(\mathrm{green}$ diamonds), $11 \mathrm{~mm}$ (black triangles up), $30 \mathrm{~mm}$ (orange triangles down). (b) Focus on the dispersion equation of the highest frequencies for the HW (red dots) and the SW (blue squares) cases: for HW the two highest frequencies are associated to localized modes. The dotted line is a guide for the eyes.

Then the MSD of the particle $n,\left\langle\Delta u(n, t)^{2}\right\rangle$, is given by

$$
\left\langle\Delta u(n, t)^{2}\right\rangle=\sum_{s=1}^{2 N-1} U_{s}(n)^{2}\left\langle\Delta X_{s}^{2}(t)\right\rangle .
$$

From Eq. (5), we recover the ballistic regime $\left\langle\Delta u(n, t)^{2}\right\rangle \stackrel{t \rightarrow 0}{\sim}$ $k_{B} T t^{2} / M$. At short times, the particles diffuse without efficiently interacting with their neighbors, nor with the confining walls. Therefore, they undergo a ballistic flight at their thermal velocity $\sqrt{k_{B} T / M}$.

In Figs. 1-3 we plot the MSD measured in the simulations and the corresponding analytical expressions obtained from Eq. (5) and Eq. (6). Although the calculations are done without any free parameter, the agreement is excellent, which shows that the dynamics of the confined particles can be explained by our model of a masses and springs chain. We will now bring some light on the qualitative behaviors of the particles by carefully studying the evolution of each normal modes according to the time.

Let us therefore discuss the variations of $\left|U_{s}(n)\right|$ with the characteristics of the confining potential $\left(\lambda_{w}, E_{w}\right)$. Figure 11 shows the variations of $\left|U_{s}(n)\right|$ according to the modes index $s$ for three different particles, the outermost one $(n= \pm 16)$, the next one $(n= \pm 15)$, and the central particle $(n=0)$ in the SW [Fig. 11(a)], HW [Fig. 11(b)], and LR [Fig. 11(c)] cases.

In cyclic systems, the amplitude of the modes does not depend on the mode index $s$ and is equal to $1 /(2 N-1)$.
Here, the amplitudes $\left|U_{s}(n)\right|$ are maximal for the modes $s$, which have an antinode at the position of the particle $n$. For a given particle $n$, the number of maxima is equal to $N-n$. This is obvious for the displacement amplitude of the central particle that is by symmetry either null (odd modes, $s$ even) or maximum (even modes, $s$ odd) according to the parity of the mode (note that the number of particles, $2 N-1$, is odd). This is indeed observed in the plots of $\left|U_{s}(0)\right|$ as a function of $s$, whatever the confinement.

For a SW confinement and a given particle $n$, the positions of the maxima look like those of an homogeneous system with a single stiffness and are roughly given by $s=\frac{N(2 q+1)}{N-n}$ where $0 \leqslant q \leqslant n$. When the wall-particle stiffness is enhanced or the confinement range extended, the amplitudes of the normal modes $\left|U_{s}(n)\right|$ are modified. As expected, the outer particles have the most important changes. For the HW case, the modes of highest amplitude are shifted towards higher frequencies. For the outermost particles $(n= \pm 16$ and $n= \pm 15$ ), the maximum amplitudes are associated to the two highest frequencies, whereas the amplitude of the center particle becomes very small for these localized modes [see Fig. 11(b)]. On the contrary, when the range of the confinement increases, the maxima of $\left|U_{s}(n)\right|$ are shifted towards lower frequencies for the two outermost particles. In this case, the contribution of the high-frequency modes to the motion of the outermost particles ( $n= \pm 16$ and $n= \pm 15)$ is extremely small [see Fig. 11(c)]. The evolution of the maxima according
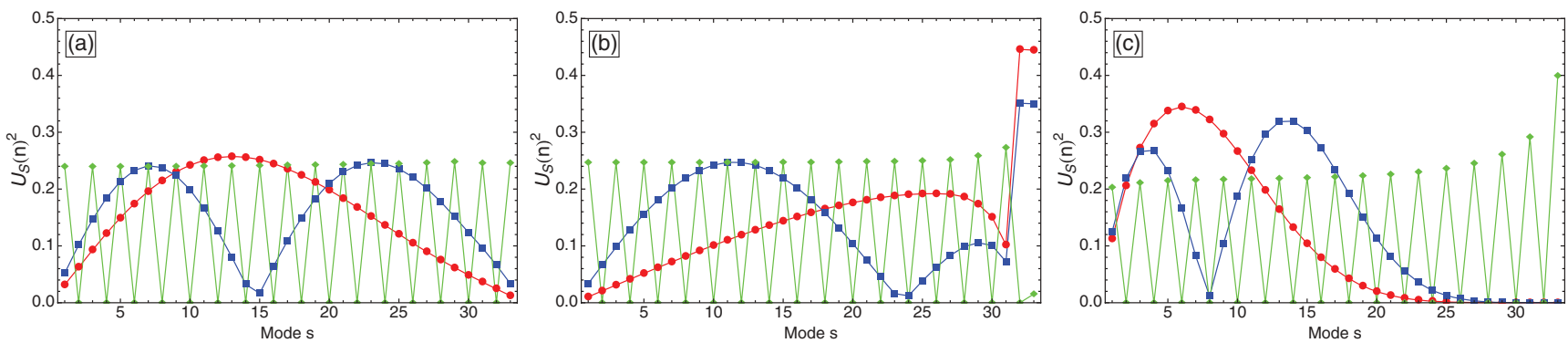

FIG. 11. (Color online) Variations of $\left|U_{s}(n)\right|$ with the mode index $s$ for three different particles, the outermost one $(n= \pm 16)$ (red dots), the next one $(n= \pm 15)$ (blue squares), and the central particle $(n=0)$ (green diamonds). (a) SW confining potential with $\lambda_{w}=1 \mathrm{~mm}$, $E_{w} / E_{0}=0.1$; (b) HW confining potential with $\lambda_{w}=0.48 \mathrm{~mm}, E_{w} / E_{0}=0.005$; (c) $\lambda_{w}=15 \mathrm{~mm}, E_{w} / E_{0}=0.1$. 

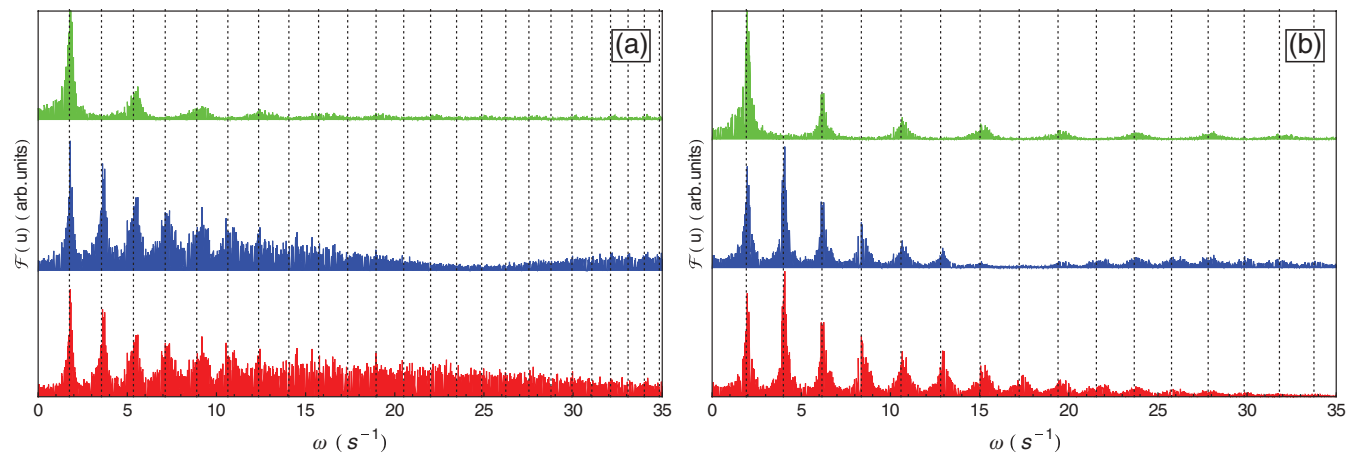

FIG. 12. (Color online) Plot of the power spectra (arb. units) of the particles trajectories as a function of the frequency $\omega\left(\mathrm{s}^{-1}\right)$ for particles $n= \pm 16$ (bottom), $n= \pm 15$ (center) and $n=0$ (top). (a) SW confinement: $\lambda_{w}=1 \mathrm{~mm}, E_{w} / E_{0}=0.1$. (b) LR confinement: $\lambda_{w}=15 \mathrm{~mm}$, $E_{w} / E_{0}=0.1$. The dotted lines indicate the first 24 (a) and 16 (b) calculated frequencies.

to the confinement is the key to understanding the diffusion behaviors of particles confined in a box. The shift towards low frequencies for the two outermost particles may be directly observed on the Fourier transform of the trajectories provided by the simulations. These Fourier transforms are shown on Fig. 12 for the particles $(n= \pm 16)$ and $(n= \pm 15)$. They exhibit indeed a shift towards low frequencies between the case of a SW confinement [Fig. 12(a)] and of a LR confinement [Fig. 12(b)].

Hereafter, we shall thus locate for each particle $n$ and each confining force $\left(\lambda_{w}, E_{w}\right)$ the local maxima associated to the mode $s_{\min }\left(s_{\max }\right)$ of smallest (highest) frequency. They will be respectively noted $s_{\min }=P_{\min }\left(n, \lambda_{w}, E_{w}\right)$ and $s_{\max }=$ $P_{\max }\left(n, \lambda_{w}, E_{w}\right)$.

\section{B. The intermediate regime}

In order to understand the intermediate regime, it is convenient to consider the contribution $\left\langle\Delta X_{s}^{2}(t)\right\rangle U_{s}(n)^{2}$ of the mode $s$ to $\left\langle\Delta u(n, t)^{2}\right\rangle$ in Eq. (6). To illustrate this point we plot in Fig. 13 the time evolution of the contributions of the modes $s=1,3$ and 33 for the center particle $(n=0)$. Two dampings are considered: $\gamma=0.1 \mathrm{~s}^{-1}$ [Fig. 13(a)] and $\gamma=60 \mathrm{~s}^{-1}$ [Fig. 13(b)]. The complete MSD curves, that include the contributions of all modes, are also displayed in order to discuss their main behaviors.

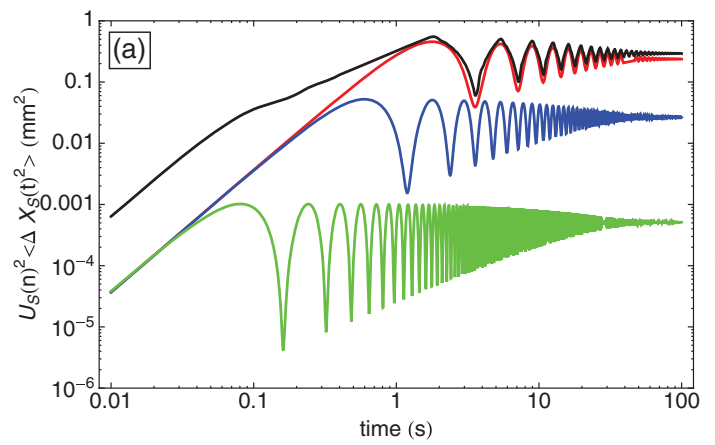

At very low damping, all modes oscillate before reaching their constant value [Fig. 13(a)]. The time evolution of $\left\langle\Delta u(n, t)^{2}\right\rangle$ in the intermediate regime is explained by the progressive disappearance in the sum (6) of the contributions of the modes $s$ as soon as they lose their initial quadratic dependence with $t$. The power series development of $\left\langle\Delta X_{s}^{2}(t)\right\rangle$ shows that it happens at a characteristic time $t \approx 2 \sqrt{3} / \omega_{s}$. Thus at a given time $t$, the variation of $\left\langle\Delta u(n, t)^{2}\right\rangle$ with time is dominated by the contributions of the modes such as $t<2 \sqrt{3} / \omega_{s}$ (strictly speaking, $t<2 \sqrt{3} / \sqrt{\omega_{s}^{2}-\gamma^{2} / 4}$, which is the same for small $\gamma$ ). The number $P(t)$ of those modes may easily be estimated in the frame of the Debye approximation

$$
P(t) \sim \frac{4 \sqrt{3} N}{\pi \sqrt{K_{D} / M} t},
$$

where $K_{D}$ is an effective stiffness such that the dispersion relation at small frequencies reads

$$
\omega_{s}=\left(\frac{\pi}{2 N} \sqrt{\frac{K_{D}}{M}}\right) s .
$$

The use of the Debye approximation is supported by the fact that the most important contributions in (6) come from the low frequencies, which follow a linear dispersion law. In the ballistic regime, each mode $s$ behaves as $\left\langle\Delta X_{s}^{2}(t)\right\rangle \sim$ $k_{B} T t^{2} / M$, and using (7) we obtain the MSD for the particle $n$

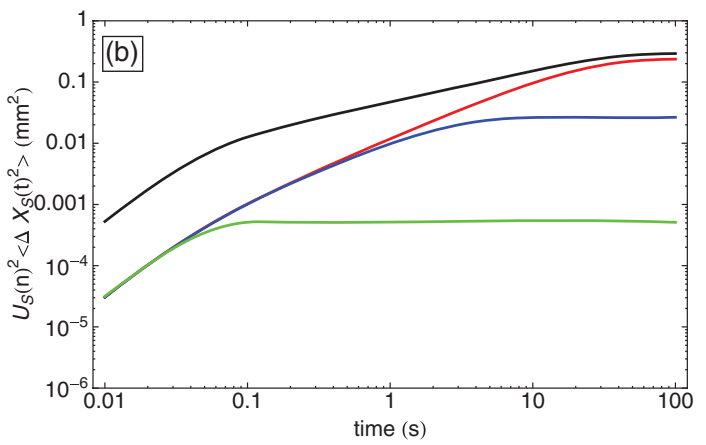

FIG. 13. (Color online) Plot of the variations with time (in s) of the contribution $\left\langle\Delta X_{s}^{2}(t)\right\rangle U_{s}(0)^{2}$ of the mode $s=1$ (red; dark gray, top), $s=3$ (blue; medium), $s=33$ (green; bottom) to the $\operatorname{MSD}\left\langle\Delta u(0, t)^{2}\right\rangle$ (in $\mathrm{mm}^{2}$ ) for a system of 33 particles and a SW confining potential with $E_{w}=0.1 E_{0}$ and $\lambda_{w}=1 \mathrm{~mm}$. (a) $\gamma=0.1 \mathrm{~s}^{-1}$, (b) $\gamma=60 \mathrm{~s}^{-1}$. In black we plot the summation on all modes. 

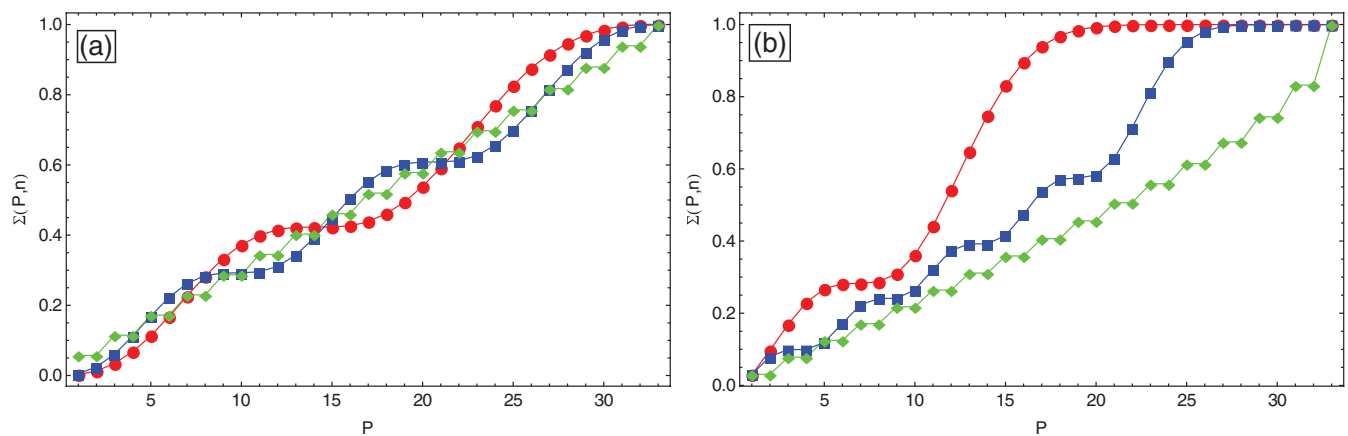

FIG. 14. (Color online) Plot of the partial sum $\Sigma(P, n)$ as a function of $P$ for particles $n= \pm 15$ (red dots), $n= \pm 14$ (blue squares), and $n=0$ (green diamonds). (a) SW confining potential with $\lambda_{w}=1 \mathrm{~mm}$ and $E_{w} / E_{0}=0.1$. (b) LR confining potential with $\lambda_{w}=30 \mathrm{~mm}$ and $E_{w} / E_{0}=0.1$.

as

$$
\left\langle\Delta u(n, t)^{2}\right\rangle \approx\left[\frac{k_{B} T}{M} \sum_{s=1}^{P(t)} U_{s}^{2}(n)\right] t^{2}=2 D t .
$$

In the opposite limit of very large damping, all modes are overdamped [Fig. 13(b)] and evolve linearly as $\left[2 k_{B} T /(M \gamma)\right] t$ before reaching saturation at a typical time $\gamma / \omega_{s}^{2}$ for the mode $s$ [13]. Now, the time evolution of $\left\langle\Delta u(n, t)^{2}\right\rangle$ is explained by the progressive disappearance in the sum (6) of the contributions of the modes $s$ when they reach their saturation. Thus, the modes that contribute to the variation of $\left\langle\Delta u(n, t)^{2}\right\rangle$ satisfy $t<\gamma / \omega_{s}^{2}$. Using once again the Debye approximation, the number $P(t)$ is estimated as

$$
P(t) \sim \frac{2 N}{\pi \sqrt{K_{D} / M \gamma} \sqrt{t}} .
$$

Using (10), the expression of the MSD of the particle $n$ is given by

$$
\left\langle\Delta u(n, t)^{2}\right\rangle \approx\left[\frac{2 k_{B} T}{M \gamma} \sum_{s=1}^{P(t)} U_{s}^{2}(n)\right] t=F \sqrt{t} .
$$

At this point, it is convenient to introduce the partial sum $\Sigma(P, n) \equiv \sum_{s=1}^{P} U_{s}^{2}(n)$ and to calculate its evolution according to the number $P$ of modes that are taken into account. Such sums associated to three different particles $n$ are presented in Fig. 14(a) and Fig. 14(b) for SR and LR confinements respectively.

In the SR case, whatever the considered particle, the sum increases roughly linearly with $P$. Then, each mode contributes to the MSD, even if they all have a different weight. We get $\Sigma(P, n)=1$ for $P=2 N-1$. Thus, for the SR case, we may write in first approximation $\Sigma(P, n) \approx$ $P /(2 N-1)$. The LR case is very different. Now, for a given particle and a given confinement, the sum is roughly linear with $P$ until $P=P_{\max }\left(n, \lambda_{w}, E_{w}\right)<(2 N-1)$ and then jumps abruptly to 1 , which means that all modes of higher index do not contribute. This is due to the smallness of amplitudes $U_{s}(n)$ associated with high-frequency modes. Thus, as long as $P<P_{\max }\left(n, \lambda_{w}, U_{w}\right)$, the sum can be written as $\Sigma(P, n)=$ $\Sigma\left(P_{\max }, n\right) P / P_{\max }$, and is equal to 1 otherwise. The evolution of $P_{\max }\left(n, \lambda_{w}, E_{w}\right)$ according to the particle index $n$ for different values of $\lambda_{w}$ is shown on Fig. 15. We see that $P_{\max }$ decreases with $|n|$.

Thus, in the case of small damping, all particles diffuse normally with $\left\langle\Delta u(n, t)^{2}\right\rangle=2 D t$ with diffusion constants $D_{\mathrm{SR}}$ and $D_{\mathrm{LR}}$ respectively associated with the SR and LR cases

$$
\begin{gathered}
D_{\mathrm{SR}}=\frac{2 \sqrt{3} k_{B} T N}{\pi(2 N-1) \sqrt{M K_{D}}}=\frac{2 \sqrt{3} k_{B} T N}{\pi(2 N-1)} \sqrt{\frac{\kappa_{T}}{M \rho}}, \\
D_{\mathrm{LR}}(n)=\frac{2 \sqrt{3} k_{B} T N}{\pi \sqrt{M K_{D}}} \frac{\Sigma\left(P_{\max }, n\right)}{P_{\max }\left(n, \lambda_{w}, E_{w}\right)} \\
=\frac{2 \sqrt{3} k_{B} T N \Sigma\left(P_{\max }, n\right)}{\pi P_{\max }\left(n, \lambda_{w}, E_{w}\right)} \sqrt{\frac{\kappa_{T}}{M \rho}},
\end{gathered}
$$

where $\rho$ is the mean particle density and $\kappa_{T}=\rho / K_{D}$ is the isothermal compressibility, which takes into account the complete distribution of the stiffnesses in the chain.

At large damping, the MSD $\left\langle\Delta u(n, t)^{2}\right\rangle$ can be expressed as $F \sqrt{t}$ where the mobilities $F$ are given, according to the confinement range, by:

$$
F_{\mathrm{SR}}=\frac{2 k_{B} T N}{\pi(2 N-1) \sqrt{M K_{D} \gamma}}=\frac{2 k_{B} T}{\pi} \frac{N}{2 N-1} \sqrt{\frac{\kappa_{T}}{M \rho \gamma}},
$$

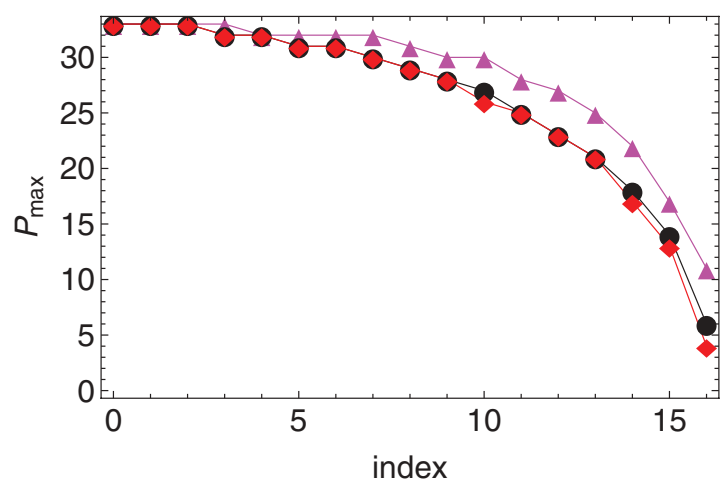

FIG. 15. (Color online) Plot of $P_{\max }\left(n, \lambda_{w}, E_{w}\right)$ as a function of the particle index $n$ for several LR confining potentials characterized by $E_{w} / E_{0}=0.1$ and $\lambda_{w}=4 \mathrm{~mm}$ (magenta triangles), $15 \mathrm{~mm}$ (black dots), and $30 \mathrm{~mm}$ (red diamonds). The values of $P_{\max }\left(16,15 \mathrm{~mm}, 0.1 E_{0}\right)$ and $P_{\max }\left(16,15 \mathrm{~mm}, 0.1 E_{0}\right)$ may be read in Fig. 11(c) 


$$
\begin{aligned}
F_{\mathrm{LR}}(n) & =\frac{4 k_{B} T N}{\pi \sqrt{M K_{D} \gamma}} \frac{\Sigma\left(P_{\max }, n\right)}{P_{\max }\left(n, \lambda_{w}, E_{w}\right)} \\
& =\frac{4 k_{B} T N}{\pi} \sqrt{\frac{\kappa_{T}}{M \rho \gamma}} \frac{\Sigma\left(P_{\max }, n\right)}{P_{\max }\left(n, \lambda_{w}, E_{w}\right)}
\end{aligned}
$$

Let us underline that the expressions $D_{\mathrm{SR}}$ and $F_{\mathrm{SR}}$ are independent of the positions of the particles. Since the only parameter that depends on the confining force is $K_{D}$, systems in which the confinement results in the same equilibrium positions of the particles have the same set of diffusivities and mobilities. Those features are displayed in the simulation results for the HW and the SW cases (see Fig. 1 and Fig. 2). In contrast, $D_{\mathrm{LR}}(n)$ and $F_{\mathrm{LR}}(n)$ explicitly depend on the index of the particles $n$ because of $P_{\max }\left(n, \lambda_{w}, E_{w}\right)$. Both increase as the particle gets closer to the walls, the highest coefficients being associated with the outermost particles. Moreover, the dependence of the diffusivities and mobilities on the effective stiffness $K_{D}$ indicates that they result from a collective effect. Lastly, note also that they are proportional, with $D / F=$ $\sqrt{\gamma / 3}$. It means that, for a given set $\left(\lambda_{w}, E_{w}\right)$, the mobility scales as $\gamma^{-1 / 2}$. This is indeed observed in the simulations, as shown in Fig. 6. As an example, for $\lambda_{w}=15 \mathrm{~mm}$ and $E_{w} / E_{0}=0.1$ the mobility is $0.07 \mathrm{~mm}^{2} \mathrm{~s}^{-1 / 2}$ for $\gamma=10 \mathrm{~s}^{-1}$ and $0.03 \approx 0.07 / \sqrt{6} \mathrm{~mm}^{2} \mathrm{~s}^{-1 / 2}$ for $\gamma=60 \mathrm{~s}^{-1}$ as indicated by Eq. (15).

In Fig. 16, the diffusivities given by the expressions (13) are plotted as a function of the index for various values of the confinement length [Fig. 16(a)] and amplitude [Fig. 16(b)]. Those values are to be compared to the simulation data presented in Fig. 4. We see that the simple estimates of Eqs. (12) and (13) catch all qualitative features of the simulation data, such as the shape of the curves and their ordering with $\lambda_{w}$ [Fig. 4(a)] and with $E_{w} / E_{0}$ [Fig. 4(b)]. Quantitatively, our estimates are in satisfactory agreement. Moreover, the mobilities may be directly calculated from the Fig. 16, multiplying by $\sqrt{\gamma / 3}$. When compared with the mobility data displayed in Fig. 6, we see that all qualitative features of the simulation data are recovered. The order of magnitude for the mobilities deduced from Fig. 16 compare well with the simulations.

\section{Crossover times}

The correlation time $t_{\text {corr }}(n)$, which characterizes the beginning of the intermediate regime of a particle $n$ is roughly given by $2 \sqrt{3} / \sqrt{\omega_{P_{\max }(n)}^{2}-\gamma^{2} / 4}$ (for the sake of readability, the dependency of $P_{\max }$ upon $\lambda_{w}$ and $E_{w}$ is not written explicitly in this section).

For the SW confinement case, most particles have $P_{\max }(n)>20$. Since at large $s$ the frequencies vary slowly with the mode number (see Fig. 10), their resulting correlation times are roughly the same. As the particle gets towards the wall, its frequency $\omega_{P_{\max }(n)}$ decreases and consequently its correlation time is somewhat longer. In the $\mathrm{HW}$ case, all the $P_{\max }(n)$ are shifted towards larger $s$, thus $t_{\text {corr }}(n)$ remains independent of the particle position but, since their frequencies are smaller than in the SW case, the resulting value of the correlation time $t_{\text {corr }}(n)$ is somewhat higher. However this concerns the inner particles only. Indeed, the frequencies associated to $P_{\max }$ for the outermost particles correspond to localized modes with higher frequencies, so these particles have a smaller $t_{\text {corr }}$.

In the LR case, all the $P_{\max }(n)$ are shifted toward the small $s$ and the high-frequency modes do not contribute efficiently to the MSD. This induces larger correlation times than in the SR cases, in particular for the outer particles for which the $P_{\max }$ are the most shifted. The evolution of the correlation times according to the particle positions for several values of $\lambda_{w}$ and $E_{w}$ are shown in Fig. 17. All these theoretical results are in good agreement with the correlation times measured from the simulations (see Fig. 7). Notice in particular that the change of evolution with $\lambda_{w}$ observed as the particle gets closer to the wall is consistent with our theoretical estimates. In the case of hard-core interactions $[6,28,29]$ this correlation time depends only on the particle concentration because the particles are free, except the direct contact with one of their neighbors. In the present situation, the particles are not actually free for $t<t_{\text {corr }}$ since they diffuse in the local potential of their neighbors and the walls, which explains why $t_{\text {corr }}$ depends on the interactions.

After the intermediate regime, the saturation time for the particle $n$ is controlled by the frequency of the mode $s=P_{\min }\left(n, \lambda_{w}, U_{w}\right)$. As before, we do not write explicitly the dependency of $P_{\min }$ upon $\lambda_{w}$ and $U_{w}$ in the rest of this section. When $\omega_{P_{\min }}>\gamma / 2$, most modes are oscillating ones as shown by Fig. 13(a). Thus, the saturation time may be estimated from Eq. (5) by $\pi\left(\omega_{P_{\min }}^{2}-\gamma^{2} / 4\right)^{-1 / 2}$. On the other hand, if
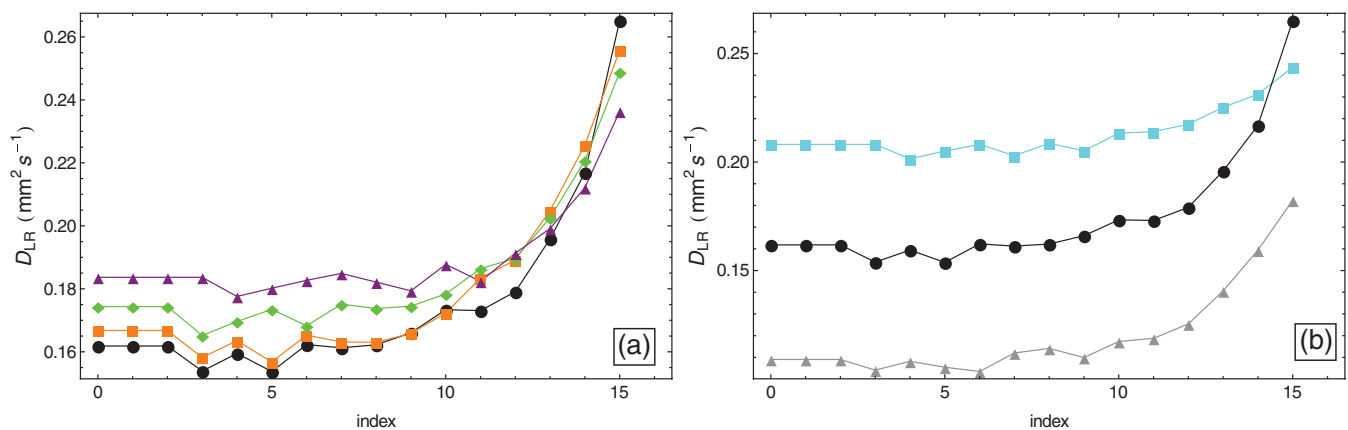

FIG. 16. (Color online) Plot of the theoretical diffusivities (in $\mathrm{mm}^{2} \mathrm{~s}^{-1}$ ) as a function of the particle index $n$. (a) $E_{w} / E_{0}=0.1, \lambda_{w}=4$ $\mathrm{mm}$ (magenta triangles), $7 \mathrm{~mm}$ (green diamonds), $11 \mathrm{~mm}$ (orange squares), and $30 \mathrm{~mm}$ (red dots). (b) $\lambda_{w}=15 \mathrm{~mm}, E_{w} / E_{0}=0.005$ (cyan squares), 0.1 (black dots), and 0.5 (gray triangles). The mobilities are $\sqrt{\gamma / 3} D_{\mathrm{LR}}$. 

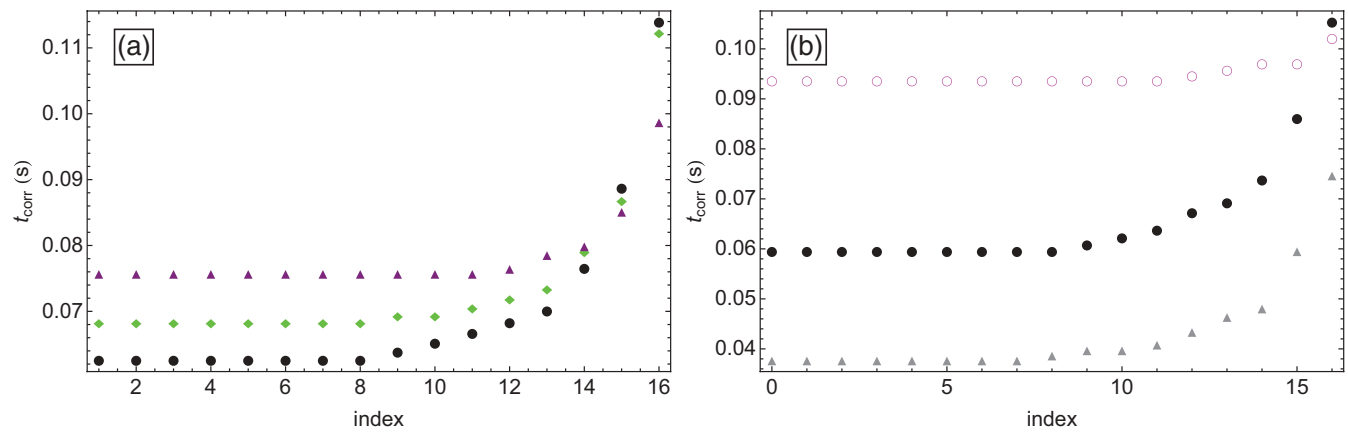

FIG. 17. (Color online) (a) Theoretical correlation times $t_{\text {corr }}$ (s) as a function of the particle index $n$. (a) $E_{w} / E_{0}=0.1 ; \lambda_{w}=4 \mathrm{~mm}$ (magenta triangles), $7 \mathrm{~mm}$ (green diamonds), $15 \mathrm{~mm}$ (black dots). (b) $\lambda_{w}=15 \mathrm{~mm} ; E_{w} / E_{0}=0.005$ (magenta circles), 0.1 (black dots), and 0.5 (gray triangles).

$\omega_{P_{\min }}<\gamma / 2$, the modes are overdamped [see Fig. 13(b)] and the saturation time is $\pi\left[\gamma / 2-\left(\omega_{P_{\min }}^{2}-\gamma^{2} / 4\right)^{1 / 2}\right]^{-1}$. At very large damping, this time is $\pi \gamma / \omega_{P_{\min }}^{2}$. At very small damping, the saturation time is $\pi / \omega_{P_{\min }}$ for all particles. Since $\omega_{P_{\min }}(n)$ is roughly equal to $\omega_{P_{\max }} N /(N-n)$, the saturation time decreases linearly with $n$. In the intermediate $\gamma$ range, the outer particles with the highest $\omega_{P_{\min }}$ could be in the small damping regime while the inner ones with smaller $\omega_{P_{\min }}$ are in the large damping regime. Thus, the saturation times are independent of $\gamma$ for the outer particles but strongly depend on the damping for the inner ones, and the evolution of $t_{\text {sat }}$ according to the index $n$ shows a jump at the particle, which corresponds to this change of regime. This analysis is in complete agreement with the behaviors observed in the simulations (see Fig. 8). The intermediate regime extends between $t_{\text {corr }}$ and $t_{\text {sat }}$, respectively, determined by the frequencies associated with $P_{\max }$ and $P_{\min }$. However, the amplitude $\left|U_{s}( \pm 16)\right|$ corresponding to the contribution of the mode $s$ to the motion of the outermost particles presents a maximum for only one mode $s$, as shown by Fig. 11 . Therefore, $t_{\text {corr }}=t_{\text {sat }}$, which explains the absence of intermediate regime for those particles.

\section{Saturation unevenness}

The behavior of the normal modes also explains the unevenness observed in the saturation regime and the evolution of its value according to $\lambda_{w}$ and $E_{w}$. This unevenness exists only when the damping is small enough for the mode $s=1$ to be an oscillating mode. It corresponds to the first local minimum in the time evolution curve of the mode $s=1$, which is typically reached at $t=2 \pi\left(\omega_{1}^{2}-\gamma^{2} / 4\right)^{-1 / 2}$. For a mode $s>1$ whose frequency is proportional to $\omega_{1}$, this time also corresponds to its $s$ th minimum (this is true at low frequencies, associated to the main contributions, since the dispersion relation is linear). The unevenness is then explained by the superposition at the same time of all these minima and its time position is independent of the particle considered. This surperposition also exists in the case of cyclic systems, but the unevenness is masked by the translationally invariant mode, which evolves linearly in time and dominates the dynamic at long time [13]. When $\lambda_{w}$ or $E_{w}$ increase, the unevenness shifts towards short times since $\omega_{1}$ increases (see Fig. 10). On the contrary, when $\gamma$ increases, the unevenness shifts towards long times. Thus, the unevenness depth $\delta_{\text {depth }}$ may be estimated as

$$
\delta_{\text {depth }} \approx \sum_{s}\left[\frac{U_{s}^{2}(n) e^{-\gamma \pi / \omega_{1}}}{\omega_{s}^{2}-\gamma^{2} / 4}\right] .
$$

It increases from the outermost particle to the central one and decreases with $\gamma$, in perfect agreement with the simulation results (see Figs. 1-3).

\section{E. Discussion}

All the behaviors obtained with these theoretical expressions are in perfect agreement with the simulation results; they also explain the experimental observations presented in Ref. [13]. The analysis of the equilibrium positions obtained in these experiments shows that the decrease of the applied voltage $V$ is equivalent to a displacement of the $\left(\lambda_{w}, E_{w}\right)$ point inside the LR domain. The observed increase of diffusivity $D$ as the voltage $V$ decreases is explained by the relation (13), as well as its increase with the particle index $|n|$. In the same way, our analysis explains the observed mobilities, which were left unexplained in Ref. [27] but are consistent with (15) (see Fig 10 of Ref. [27]. There is a misprint in the caption where the colors have been transposed. The text in the body of the paper is correct). In this article, we also presented a surprising result concerning the correlation time. While the correlation time measured for $V=1300 \mathrm{~V}$ is always higher than the one measured at $1000 \mathrm{~V}$, the opposite is observed for the outermost balls (see Fig 8. of Ref. [27]). This is in agreement with our estimations of the correlation time displayed in Fig. 17.

\section{CONCLUSION}

We have described the SFD of a finite number of particles with soft-core interactions, confined in a linear and finite box and submitted to thermal fluctuations. We have performed numerical simulations of the relevant Langevin equations and proposed to describe this system as a chain of linear springs and point masses in a thermal bath. In a previous work [30], we have identified three different kinds of confinement, according to the relative values of the springs: The SR confining forces are such that interparticle interactions may be described by identical springs, with HW and SW confining forces corresponding to a wall-particle interaction respectively higher and smaller than the interparticle interactions. For the 
LR confinement the springs vary along the line. Since such systems have finite size, the particles' MSD reach constant values at very long times $\left(t_{\mathrm{sat}} \leqslant t\right)$. In a previous article, we have studied the effects of the confinement on the MSD in this saturation regime [30]. Here we focus on the transient behaviors at short times.

Two regimes have been identified for the transient time evolution of the MSD $\left\langle\Delta u(n, t)^{2}\right\rangle$ of the $n$th particle. At small times $\left(0 \leqslant t \leqslant t_{\text {corr }}\right)$, it evolves as $\left\langle\Delta u(n, t)^{2}\right\rangle=\left(k_{B} T / m\right) t^{2}$. This is a ballistic flight that results from the inertial effects and is observed whatever the damping $\gamma$, the particle $n$, and the confinement range. For $\left(t_{\text {corr }} \leqslant t \leqslant t_{\text {sat }}\right)$, an intermediate regime takes place in which the power law describing the evolution of the MSD according to the time depends on the damping value. For small damping, a linear scaling in $t$ is observed and a constant diffusivity may be defined for all the particles. For large damping, the MSD undergoes a SFD scaling in $t^{1 / 2}$ and mobilities may be determined. In these finite-size linear systems, the translation invariance is broken and the evolution of the MSD according to the time depends on the rank of the particle in the line.

We exhibit a strong dependency of the dynamics on the confinement. While the dynamic of the outermost particles is the slowest in the HW case, it is by contrast the fastest in the SW and LR cases. Moreover, while the behavior of the MSD may be described by a single diffusivity or a single mobility in the case of a SR confinement, these coefficients vary along the line for a LR confinement. In this case, both coefficients increase when the considered particle is far from the confining walls. The crossover times depend on the confinement too. For LR confinement, the correlation times strongly increase for particles close to the wall whereas the saturation times decrease as the particle gets farther from the walls.

From a theoretical point of view, we show that the motion of the particles of such systems may be described in terms of the normal modes of oscillation of the corresponding chain of springs and point masses. The MSD results from the superposition of all these modes, which undergo the same dynamic as an oscillator in an harmonic well. The overdamped modes, which do not oscillate until they saturate, contribute to the SFD scaling in $t^{1 / 2}$. The underdamped modes oscillate before reaching their saturation and contribute to the linear time scaling. The particularity of finite-sized linear systems is that the particles are not equivalent. Each of them is associated with specific modes in which they oscillate preferentially. Thus, the superposition of modes is different for each particle. Complete expressions of their diffusivities and mobilities have been expressed according to the relevant parameters. Those estimates are in perfect agreement with our simulation data. With this model, we recover the correct power laws for the time dependency of the MSD in the various regimes, and we are able to estimate the prefactors according to the relevant parameters.
[1] D. Jepsen, J. Math. Phys. 6, 405 (1965).

[2] R. Arratia, Ann. Prob. 11, 362 (1983).

[3] P.-G. de Gennes, J. Chem. Phys. 55, 572 (1971).

[4] D. G. Levitt, Phys. Rev. A 8, 3050 (1973).

[5] P. M. Richards, Phys. Rev. B 16, 1393 (1977).

[6] H. van Beijeren, K. W. Kehr, and R. Kutner, Phys. Rev. B 28, 5711 (1983).

[7] J. Kärger, Phys. Rev. A 45, 4173 (1992).

[8] K. Hahn and J. Kärger, J. Phys. A 28, 3061 (1995).

[9] K. Hahn and J. Kärger, J. Phys. Chem. B 102, 5766 (1998).

[10] A. Taloni and F. Marchesoni, Phys. Rev. E 74, 051119 (2006).

[11] M. Kollmann, Phys. Rev. Lett. 90, 180602 (2003).

[12] B. Felderhof, J. Chem. Phys. 131, 064504 (2009).

[13] J.-B. Delfau, C. Coste, and M. Saint Jean, Phys. Rev. E 84, 011101 (2011).

[14] T. Ooshida, S. Goto, T. Matsumoto, A. Nakahara, and M. Otsuki, J. Phys. Soc. Jpn. 80, 074007 (2011).

[15] B. Lin, M. Meron, B. Cui, S. A. Rice, and H. Diamant, Phys. Rev. Lett. 94, 216001 (2005).

[16] V. Borman, B. Johansson, N. Skorodumova, I. Tronin, V. Tronin, and V. Troyan, Phys. Lett. A 359, 504 (2006).

[17] Q.-H. Wei, C. Bechinger, and P. Leiderer, Science 287, 625 (2000).

[18] B. Lin, B. Cui, J.-H. Lee, and J. Yu, Europhys. Lett. 57, 724 (2002).

[19] C. Lutz, M. Kollmann, P. Leiderer, and C. Bechinger, J. Phys.: Condens. Matter 16, S4075 (2004).

[20] G. Coupier, C. Guthmann, Y. Noat, and M. Saint Jean, Phys. Rev. E 71, 046105 (2005).
[21] G. Coupier, M. Saint Jean, and C. Guthmann, Phys. Rev. E 73, 031112 (2006).

[22] G. Coupier, M. Saint Jean, and C. Guthmann, Europhys. Lett. 77, 60001 (2007).

[23] C. Coste, J.-B. Delfau, C. Even, and M. Saint Jean, Phys. Rev. E 81, 051201 (2010).

[24] K. Nelissen, V. Misko, and F. Peeters, Europhys. Lett. 80, 56004 (2007).

[25] S. Herrera-Velarde and R. Castañeda-Priego, J. Phys.: Condens. Matter 19, 226215 (2007).

[26] L. Lizana, T. Ambjornsson, A. Taloni, E. Barkai, and M. A. Lomholt, Phys. Rev. E 81, 051118 (2010).

[27] J.-B. D. Delfau, C. Coste, C. Even, and M. Saint Jean, Phys. Rev. E 82, 031201 (2010).

[28] L. Lizana and T. Ambjörnsson, Phys. Rev. Lett. 100, 200601 (2008).

[29] L. Lizana and T. Ambjörnsson, Phys. Rev. E 80, 051103 (2009).

[30] J.-B. Delfau, C. Coste, and M. Saint Jean, Phys. Rev. E 85, 041137 (2012).

[31] S. Herrera-Velarde and R. Castañeda-Priego, Phys. Rev. E 77, 041407 (2008).

[32] B. Cui, H. Diamant, and B. Lin, Phys. Rev. Lett. 89, 188302 (2002).

[33] C. Lutz, M. Kollmann, and C. Bechinger, Phys. Rev. Lett. 93, 026001 (2004).

[34] M. Köppl, P. Henseler, A. Erbe, P. Nielaba, and P. Leiderer, Phys. Rev. Lett. 97, 208302 (2006).

[35] P. Henseler, A. Erbe, M. Köppl, P. Leiderer, and P. Nielaba, Phys. Rev. E 81, 041402 (2010). 